Bull. Soc. math. France

134 (3), 2006, p. 327-355

\title{
DISCRÉTISATION DE ZETA-DÉTERMINANTS D'OPÉRATEURS DE SCHRÖDINGER SUR LE TORE
}

\author{
par Laurent Chaumard
}

\begin{abstract}
RÉSumÉ. - Nous donnons ici deux résultats sur le déterminant $\zeta$-régularisé $\operatorname{det}_{\zeta} A$ d'un opérateur de Schrödinger $A=\Delta_{g}+V$ sur une variété compacte $\mathcal{M}$. Nous construisons, pour $\mathcal{M}=S^{1} \times S^{1}$, une suite $\left(G_{n}, \rho_{n}, \Delta_{n}\right)$ où $G_{n}$ est un graphe fini qui se plonge dans $\mathcal{M}$ via $\rho_{n}$ de telle manière que $\rho_{n}\left(G_{n}\right)$ soit une triangulation de $\mathcal{M}$ et où $\Delta_{n}$ est un laplacien discret sur $G_{n}$ tel que pour tout potentiel $V \operatorname{sur} \mathcal{M}$, la suite de réels $\operatorname{det}\left(\Delta_{n}+V\right)$ converge après renormalisation vers $\operatorname{det}_{\zeta}\left(\Delta_{g}+V\right)$. Enfin, nous donnons sur toute variété riemannienne compacte $(\mathcal{M}, g)$ de dimension inférieure ou égale à 3 et de groupe d'isométries transitif, un majorant du déterminant $\operatorname{det}_{\zeta}\left(\Delta_{g}+V\right)$, lorsque le potentiel $V$ est positif.

ABSTRACT (Discretisation of Schrodinger operators on torus). - We propose two results concerning the $\zeta$-regularised determinant $\operatorname{det}_{\zeta} A$ of a Schrödinger operator $A=\Delta_{g}+V$ on a compact riemannian manifold $(\mathcal{M}, g)$. For $\mathcal{M}=S^{1} \times S^{1}$, we construct a sequence $\left(G_{n}, \rho_{n}, \Delta_{n}\right)$ where $G_{n}$ is a finite graph injected in $\mathcal{M}$ via $\rho_{n}$, in such a way that $\rho_{n}\left(G_{n}\right)$ triangulates $\mathcal{M} . \Delta_{n}$ is a discrete laplacian on $G_{n}$ so that for every potential $V$ on $\mathcal{M}$, the sequence $\operatorname{det}\left(\Delta_{n}+V\right)$ converges, after normalisation, to $\operatorname{det}_{\zeta}\left(\Delta_{g}+V\right)$. Last, we give on every riemannian compact manifold $(\mathcal{M}, g)$ whose dimension is less than or equal to 3 and with a transitiv isometry group, the maximum of the determinant $\operatorname{det}_{\zeta}\left(\Delta_{g}+V\right)$.
\end{abstract}

Texte reçu le 19 octobre 2004, accepté le 11 avril 2005.

Laurent Chaumard, Lycée Joffre, 150, allée de la Citadelle, 34000 Montpellier (France). E-mail : chaumard@no-log.org

Classification mathématique par sujets (2000). — 53B21, 53C24,94C15, 53A35, 58J40, 58J50.

Mots clefs. - Déterminant zeta-régularisé, théorie spectrale des graphes et des surfaces, discrétisation, fonction zeta, opérateur de Schrödinger, opérateurs pseudo-différentiels, géométrie Riemannienne. 


\section{Introduction}

Dans les années 1970, D.B. Ray et I.M. Singer [14] ont proposé une approche originale de l'étude du spectre des opérateurs de Schrödinger sur une variété compacte, en définissant une fonctionnelle $\operatorname{det}_{\zeta}$, régularisation du produit des valeurs propres d'un tel opérateur $A$, par la formule

$$
\operatorname{det}_{\zeta} A:=\exp \left\{-\frac{\mathrm{d}}{\mathrm{d} s}{ }_{s=0} \zeta_{A}(s)\right\} \quad \text { où } \quad \zeta_{A}(s):=\operatorname{Trace}_{L_{2}} A^{-s} .
$$

Cet invariant spectral s'avèrera rapidement non trivial, et B. Osgood, R. Phillips, P.Sarnak [12] mettront en lumière l'information géométrique qu'il contient en prouvant qu'il fournit une nouvelle démonstration du théorème d'uniformisation des surfaces, ainsi que des résultats de pré-compacité d'ensembles isospectraux de métriques en dimension 2 [11].

M. Pollicott, A.C. Rocha [13] établiront ensuite une expression, pour des mé-

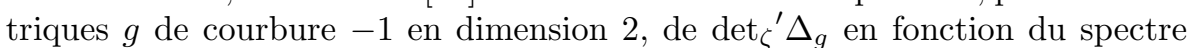
des longueurs de $g$, mettant ainsi en exergue les qualités dynamiques du déterminant. Plus récemment, K. Okikiolu [10] a obtenu, en dimension impaire, des formules de variations premières et secondes en fonction du facteur conforme de la métrique, du déterminant du laplacien conforme.

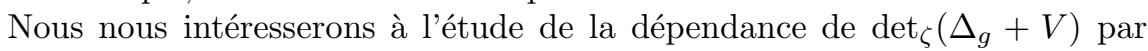
rapport au potentiel $V$, en dimension 2 ou 3, via deux approches distinctes.

\section{Maximum $\operatorname{de}_{\operatorname{det}}\left(\Delta_{g}+V\right)$}

Le premier résultat établit sous certaines conditions topologiques et géomé-

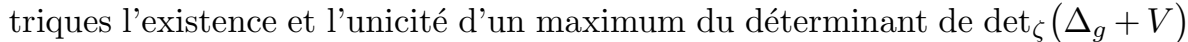
lorsque $V$ varie.

THÉORÈme 1.1. - Soit $\left(\mathcal{M}^{m}, g\right)$ une variété riemannienne compacte sans bord. Soit $V \in C^{\infty}\left(\mathcal{M}, \mathbb{R}^{+}\right)$un potentiel non nul. Posons

Alors :

$$
v_{0}:=\frac{1}{\operatorname{Vol}_{g}(\mathcal{M})} \int_{\mathcal{M}} V \mathrm{~d} v_{g}
$$

1) Si $m \leq 3$ et le groupe d'isométries de g agit de manière transitive sur $\mathcal{M}$, alors on a

$$
\operatorname{det}_{\zeta}\left(\Delta_{g}+V\right) \leq \operatorname{det}_{\zeta}\left(\Delta_{g}+v_{0}\right)
$$

avec égalité si et seulement si $V$ est un potentiel constant.

2) Si $\mathcal{M}=S^{2}$ ou $S^{1} \times S^{1}$, soit $\varphi: \mathcal{M} \mapsto \mathbb{R}$, telle que la métrique $\mathrm{e}^{2 \varphi} \mathrm{g}$ soit de courbure constante et de même volume que $\mathrm{g}$. Nous avons l'inégalité

$$
F_{g}(V) \leq F_{g}\left(v_{0} \mathrm{e}^{2 \varphi}\right) \text { ò̀ } \quad F_{g}(V):=\exp \left\{\frac{1}{2 \pi} \int_{\mathcal{M}} \varphi V \mathrm{~d}_{g}\right\} \operatorname{det}_{\zeta}\left(\Delta_{g}+V\right),
$$

TOME $134-2006-\mathrm{N}^{\mathrm{O}} 3$ 
le cas d'égalité n'étant réalisé que si le potentiel $V \mathrm{e}^{-2 \varphi}$ est constant.

L'idée de la démonstration est relativement simple; il s'agira de prouver que sous nos hypothèses topologiques, le déterminant est une fonctionnelle logconcave du potentiel et que pour les métriques étudiées dans le premier point, le noyau de la différentielle du logarithme du déterminant calculée en un potentiel constant est l'hyperplan des potentiels d'intégrale nulle. Le cas général sera obtenu par une généralisation de la formule de Polyakov.

On remarque en particulier que sous les hypothèses métriques du cas 1), les potentiels constants sont spectralement rigides. Enfin, en dimension inférieure à 3 , à cause de la stricte log-concavité, et indépendamment de la métrique, il n'existe aucune déformation isospectrale $C^{\infty}$ de potentiels.

\section{Des déterminants de graphe vers $\operatorname{det}_{\zeta}$}

L'approche discrète de l'invariant spectral $\operatorname{det}_{\zeta}$ prend tout son sens à la lumière à la fois des liens forts qu'il existe entre les quantités spectrales des graphes et celles des variétés, et de la simplicité, au moins dans sa définition, du déterminant d'un opérateur de Schrödinger discret. Précisément, il serait intéressant d'établir, pour un opérateur de Schrödinger $A$ sur une variété compacte $\mathcal{M}$, s'il existe une suite de graphes $\left(G_{n}\right)_{n \in \mathbb{N}}$, de sommets notés $\left(S_{n}\right)_{n \in \mathbb{N}}$, et d'opérateurs de Schrödinger $A_{n}: \mathbb{R}^{S_{n}} \rightarrow \mathbb{R}^{S_{n}}$, de préférence explicites, tels que la suite $\left(\operatorname{det} A_{n}\right)_{n \in \mathbb{N}}$ converge vers $\operatorname{det}_{\zeta} A$.

Ce problème a été résolu en dimension 1 par D. Burgheela, L. Friedlander, T. Kappeler [1], R. Forman [7] et Y. Colin De Verdière [4]. Notons $\mathcal{M}=[0, T]$ et $G_{n}$ le graphe linéaire à $n$ sommets vu comme une discrétisation de $\mathcal{M}$. Soit un opérateur de Sturm-Liouville $A=-\frac{\mathrm{d}^{2}}{\mathrm{~d} t^{2}}+V(t)$ sur $[0, T]$, auquel on associe, par la méthode des éléments finis la plus naturelle qui soit, une suite d'opérateurs différentiels discrets $A_{n}: \mathbb{R}^{S_{n}} \rightarrow \mathbb{R}^{S_{n}}$. Les auteurs ci-dessus montrent qu'après normalisation, les déterminants discrets convergent vers $\operatorname{det}_{\zeta} A$ :

THÉORÈme 1.2 (cf. [1], [7] et [4]). — Il existe une constante c, ne dépendant que des conditions au bord, telle que

$$
\frac{\operatorname{det} A_{n}}{n^{2 n}} \underset{n \rightarrow \infty}{\longrightarrow} c \operatorname{det}_{\zeta} A .
$$

Plus précisément, est prouvée la convergence vers le déterminant de Feynman de $A$, défini par

$$
\operatorname{det}_{F} A:=\operatorname{det}\left(v \in \mathbb{R}^{p} \rightarrow Y_{v}(T)\right),
$$

où $Y_{v}:[0, T] \rightarrow \mathbb{R}^{p}$ est l'unique fonction vérifiant

$$
A\left(Y_{v}\right)=0 \operatorname{sur}[0, T], \quad Y_{v}(0)=0, \quad Y_{v}^{\prime}(0)=v .
$$

La proportionnalité entre le déterminant de Feynman et le déterminant $\zeta$ régularisé est démontrée dans [7]. Cette formule s'avère à la fois étonnante et très utile, puisqu'elle permet de contourner l'obstacle majeur dans l'étude de

BULlETIN DE LA SOCIÉTÉ MATHÉMATIQUE DE FRANCE 
$\operatorname{det}_{\zeta}$, à savoir la régularisation nécessaire à sa définition. En effet, $\operatorname{det}_{F}$ n'est finalement que le déterminant d'une matrice d'ordre $p$.

Notons que les auteurs des articles [1] et [4] prouvent que dans le cas des graphes linéaires, le déterminant de $A_{n}$ est également proportionnel à la valeur au bord d'un vecteur du noyau de $A_{n}$, pendant discret de (2). Après le calcul de ce facteur de proportionnalité, ils concluent en utilisant le fait qu'une approximation du type Euler de cette équation différentielle converge vers la solution continue. R. Forman [7] prouve quant à lui indépendamment de (2) que det $A_{n} / n^{2 n}$ converge vers le déterminant $\zeta$-régularisé.

Nous proposons ici un résultat similaire en dimension 2.

ThÉorÈme 1.3. - Soit $\mathcal{M}=S^{1} \times S^{1}$ le tore de dimension 2 muni d'une métrique riemannienne $g$. Alors, il existe

- une suite de triangulations $\left(G_{n}\right)_{n \in \mathbb{N}}=\left(S_{n}, E_{n}\right)_{n \in \mathbb{N}}$ de $\mathcal{M}$ ne dépendant que de la classe conforme de $g$,

- une suite d'opérateurs laplaciens explicites $\left(\Delta_{n}: \mathbb{R}^{S_{n}} \rightarrow \mathbb{R}^{S_{n}}\right)_{n \in \mathbb{N}}$, discrétisés de $\Delta_{g}$ par une méthode des éléments finis,

telles que pour toute fonction $C^{\infty}$ positive $V$ non identiquement nulle sur $\mathcal{M}$,

$$
\frac{\operatorname{det}\left(\Delta_{n}+V\right)}{\operatorname{det}\left(\Delta_{n}+v_{0}\right)} \underset{n \rightarrow \infty}{\longrightarrow} \frac{\operatorname{det}_{\zeta}\left(\Delta_{g}+V\right)}{\operatorname{det}_{\zeta}\left(\Delta_{g}+v_{0}\right)} \exp \left\{-\frac{1}{2 \pi} \int_{\mathcal{M}} K_{g} \Delta_{g}^{-1}\left(V-v_{0}\right) \mathrm{d} v_{g}\right\},
$$
où $v_{0}:=1 / \operatorname{Vol}_{g}(\mathcal{M}) \int_{\mathcal{M}} V \mathrm{~d} v_{g}$ et $K_{g}: \mathcal{M} \rightarrow \mathbb{R}$ est la courbure de Gauss de $g$.

Le schéma de preuve reprend l'approche variationnelle de [7]. Nous utiliserons les formules sur la différentielle du déterminant des opérateurs de Schrödinger obtenus dans la partie 2. Posons pour tout $t$ dans $[0,1]$,

$$
A_{t}:=\Delta_{g}+v_{0}+V_{t}, \quad \text { où } \quad V_{t}:=t\left(V-v_{0}\right),
$$

et définissons, pour chaque $t,\left(A_{t, n}\right)_{n \geq 3}$ la suite des discrétisés de $A_{t}$. Soient $\psi_{n}$ et $\psi$ les fonctions $C^{\infty}$ définies sur un ouvert de $\mathbb{R}$ contenant $[0,1]$ par

$$
\psi_{n}(t):=\ln \operatorname{det} A_{t, n} \quad \text { et } \quad \psi(t):=\ln \operatorname{det}_{\zeta} A_{t} .
$$

R. Forman prouve que la suite $\left(\psi_{n}^{\prime}\right)$ converge uniformément vers $\psi^{\prime}$ sur $[0,1]$. Il dispose pour cela d'une formule de variation première qui, pour des raisons que nous verrons par la suite, n'est plus valable en dimension 2. C'est pourquoi nous nous intéresserons aux dérivées secondes. Dans le cas où la courbure de $g$ est nulle, le théorème 1.3 est ainsi une conséquence de la convergence uniforme de $\psi_{n}^{\prime}$ vers $\psi^{\prime}$ sur $[0,1]$, qui sera obtenue à partir des deux propriétés suivantes :

$$
\sup _{[0,1]}\left|\psi_{n}^{\prime \prime}-\psi^{\prime \prime}\right| \underset{n \rightarrow \infty}{\longrightarrow} 0 \quad \text { et } \quad \psi_{n}^{\prime}(0) \underset{n \rightarrow \infty}{\longrightarrow} \psi^{\prime}(0) .
$$

Pour une métrique $g$ quelconque sur $\mathcal{M}$, nous nous ramènerons au cas précédent grâce au théorème d'uniformisation des surfaces et au corollaire 3.2, généralisation de la formule de Polyakov.

TOME $134-2006-\mathrm{N}^{\mathrm{O}} 3$ 
Dans la partie 2, nous démontrons une formule de variation seconde du logarithme du déterminant. Dans la partie 3, nous démontrons le théorème 1.1, et dans la dernière partie le théorème 1.3.

\section{Les dérivées secondes du déterminant}

Dans cette partie, $(\mathcal{M}, g)$ sera une variété riemannienne compacte sans bord de dimension $m, V$ sera un potentiel positif non identiquement nul et nous poserons

$$
v_{0}:=\frac{1}{\operatorname{Vol}_{g}(\mathcal{M})} \int_{\mathcal{M}} V \mathrm{~d} v_{g}
$$

Enfin, pour tout $t \in[0,1]$, nous considèrerons l'opérateur de Schrödinger

$$
A_{t}:=\Delta_{g}+V_{t}
$$

où $V_{t}:=v_{0}+t\left(V-v_{0}\right)$. Nous notons $I$ un ouvert de $\mathbb{R}$ contenant $[0,1]$ tel que $A_{t}$ est injectif pour tout $t \in I$. L'opérateur dérivé de $A_{t}$ sera noté

$$
\mathcal{V}: f \in L^{2}(\mathcal{M}) \longmapsto\left(V-v_{0}\right) f \in L^{2}(\mathcal{M}) .
$$

Lemme 2.1. - Pour tout complexe $z$ et tout $t_{0} \in I$, l'opérateur $\left.\frac{\mathrm{d}}{\mathrm{d} t}\right|_{t=t_{0}}\left(A_{t}^{z}\right)$ est un opérateur pseudo-différentiel d'ordre $2 p-2$ pour tout $p$ strictement plus grand que la partie réelle de z.

Démonstration. - Plaçons-nous dans une carte de la variété afin de nous ramener à un ouvert de $\mathbb{R}^{m}$. Si l'on note $a_{0}, a_{1}$ et $a_{2}$ les trois composantes homogènes en $\left(\xi, \lambda^{\frac{1}{2}}\right)$ du symbole total de $A-\lambda \mathrm{Id}$, nous avons

$$
\begin{aligned}
& a_{2}(x, \xi, \lambda)=\sum_{i, j=1}^{m} g^{i j}(x) \xi_{i} \xi_{j}-\lambda, \\
& a_{1}(x, \xi, \lambda)=-\frac{1}{\sqrt{G}} \sum_{i, j=1}^{m} \xi_{j} \mathrm{~d}_{x}\left(g^{i j} \sqrt{G}\right) \cdot \xi_{i}, \\
& a_{0}(x, \xi, \lambda)=v_{0}+t V_{1}(x)=v_{0}+t\left(V(x)-v_{0}\right),
\end{aligned}
$$

où $\left(g^{i j}(x)\right)_{1 \leq i, j \leq m}$ est la matrice de la métrique induite par $g$ sur $\left(T_{x} \mathcal{M}\right)^{*}$, lue dans la carte, et $G=\operatorname{det} g^{i j}$.

Notons maintenant $b_{-2-j, t}^{0}(x, \xi, \lambda)$, pour $j=0,1,2, \ldots$, les composantes homogènes d'un développement asymptotique du symbole d'un paramétrix de $A_{t}-\lambda$ Id (le premier indice étant le degré d'homogénéité de ce coefficient en 
$\left(\xi, \lambda^{\frac{1}{2}}\right)$, voir [15]). Alors, pour $\|\xi\|$ suffisamment grand,

$$
\begin{aligned}
b_{-2, t}^{0}(x, \xi, \lambda) & =\left(a_{2}(x, \xi, \lambda)\right)^{-1}, \\
b_{-3, t}^{0}(x, \xi, \lambda) & =-\left(a_{2}(x, \xi, \lambda)\right)^{-1}\left[a_{1}(x, \xi, \lambda) b_{-2, t}^{0}(x, \xi, \lambda)\right. \\
& \left.\quad+\sum_{i=1}^{m} \partial_{\xi_{i}} a_{2}(x, \xi, \lambda) D_{x_{i}} b_{-2, t}^{0}(x, \xi, \lambda)\right]
\end{aligned}
$$

ne dépendent donc pas de $t$, tout comme les deux premières composantes homogènes $b_{2 z, t}^{(z), 0}(x, \xi)$ et $b_{2 z-1, t}^{(z), 0}(x, \xi)$ du symbole de $A_{t}^{z}$, en vertu des relations (voir [15]) :

$$
b_{2 z-j, t}^{(z), 0}(x, \xi)=\frac{i}{\pi} \int_{\Gamma} \lambda^{z} b_{-2-j, t}^{0}(x, \xi, \lambda) \mathrm{d} \lambda,
$$

où $\Gamma$ est un contour infini dans $\mathbb{C}$ convenablement choisi. En dérivant par rapport à $t$ le symbole de $A_{t}^{z}$, ces deux termes disparaissent donc, et nous obtenons ainsi le résultat du lemme.

Il existe une formule exprimant la variation première du logarithme du déterminant en termes de fonctions $\zeta$ (voir [2]).

ThÉorème 2.2 (D. Burgheela, L. Friedlander et T. Kappeler)

La fonction $t \in I \mapsto \operatorname{det}_{\zeta} A_{t}$ est une fonction lisse et

$$
\frac{\mathrm{d}}{\mathrm{d} t} \ln \operatorname{det}_{\zeta} A_{t}=\mathrm{FP}_{s=0} \operatorname{Trace}_{L_{2}}\left(\mathcal{V} \circ A_{t}^{-1-s}\right),
$$

où $\mathrm{FP}_{s=0}\left(a_{-1} / s+a_{0}+a_{1} s+\cdots\right):=a_{0}$ et $\mathcal{V}$ est l'opérateur de multiplication par le potentiel $V-v_{0}$.

Nous en dérivons une formule de variation seconde :

Proposition 2.3. - Si $\mathcal{M}$ est de dimension $m \leq 3$, alors pour tout réel $t \in I$,

$$
\frac{\mathrm{d}^{2}}{\mathrm{~d} t^{2}} \ln \operatorname{det}_{\zeta} A_{t}=-\operatorname{Trace}_{L_{2}}\left(\mathcal{V} \circ A_{t}^{-1} \circ \mathcal{V} \circ A_{t}^{-1}\right) \text {. }
$$

En particulier, $t \mapsto \ln \operatorname{det}_{\zeta} A_{t}$ est strictement concave si $V$ n'est pas un potentiel constant.

Démonstration. - On a

$$
\begin{aligned}
\frac{\mathrm{d}^{2}}{\mathrm{~d} t^{2}} \ln \operatorname{det}_{\zeta}\left(\Delta_{g}+v_{0}+t V_{1}\right)= & \frac{\mathrm{d}}{\mathrm{d} t}[ \\
= & \operatorname{FP}_{s=0} \operatorname{Trace}_{L_{2}} \mathcal{V} \circ\left(\frac{\mathrm{d}}{\mathrm{d} t}\left(A_{t}^{-1}\right)\right) \circ A_{t}^{-s} \\
& \quad+\operatorname{FP}_{s=0} \operatorname{Trace}_{L_{2}} \mathcal{V} \circ A_{t}^{-1} \circ \frac{\mathrm{d}}{\mathrm{d} t}\left(A_{t}^{-s}\right) .
\end{aligned}
$$

TOME $134-2006-\mathrm{N}^{\mathrm{O}} 3$ 
Or $\mathcal{V} \circ\left(\frac{\mathrm{d}}{\mathrm{d} t}\left(A_{t}^{-1}\right)\right) \circ A_{t}^{-s}=-\mathcal{V} \circ A_{t}^{-1} \circ \mathcal{V} \circ A_{t}^{-1-s}$ est un opérateur pseudodifférentiel à trace au voisinage de $s=0$, et donc

$$
\mathrm{FP}_{s=0} \operatorname{Trace}_{L_{2}}\left[\mathcal{V} \circ\left(\frac{\mathrm{d}}{\mathrm{d} t}\left(A_{t}^{-1}\right)\right) \circ A_{t}^{-s}\right]=-\operatorname{Trace}_{L_{2}}\left[\mathcal{V} \circ A_{t}^{-1} \circ \mathcal{V} \circ A_{t}^{-1}\right]
$$

Quant au deuxième terme, il s'annule car d'après le lemme 2.1, l'opérateur $\frac{\mathrm{d}}{\mathrm{d} t}\left(A_{t}^{-s}\right)$ est un opérateur pseudo-différentiel à trace au voisinage de $s=0$ et indépendant de $t$ si $s=0$.

Enfin, la concavité vient du fait que, écrite dans une base hilbertienne de fonctions propres de $A_{t}$, la trace est une somme de termes strictement positifs.

\section{Le théorème 1.1}

Nous avons besoin d'un dernier résultat avant de démontrer le théorème 1.1. Il s'agit d'une adaptation de la formule de Polyakov au cas des opérateurs de Schrödinger. Précisément, nous avons :

Proposition 3.1. - Soit $\left(\mathcal{M}, g_{0}\right)$ une surface riemannienne compacte sans bord. Soit $V$ un potentiel $C^{\infty}$ positif non nul sur $\mathcal{M}$ et $\varphi$ une fonction $C^{\infty}$ sur $\mathcal{M}$. Alors, si $A:=\Delta_{g_{0}}+V$, nous avons la relation

(4) $\ln \frac{\operatorname{det}_{\zeta} \mathrm{e}^{-2 \varphi} A}{\operatorname{det}_{\zeta} A}=-\frac{1}{6 \pi}\left[\frac{1}{2} \int_{\mathcal{M}}\left|\nabla_{0} \varphi\right|^{2} \mathrm{~d} v_{0}+\int_{\mathcal{M}} K_{0} \varphi \mathrm{d} v_{0}-3 \int_{\mathcal{M}} V \varphi \mathrm{d} v_{0}\right]$,

où $\mathrm{d} v_{0}$ est la mesure volume de la métrique $g_{0}, K_{0}$ sa courbure de Gauss, et $\int_{\mathcal{M}}\left|\nabla_{0} \varphi\right|^{2} \mathrm{~d} v_{0}$ l'énergie de Dirichlet de $\varphi$ pour la métrique $g_{0}$.

La démonstration est identique à celle apparaissant dans l'article de [12], à ceci près que si on note $\mathrm{d} v$ la mesure volume de $g=\mathrm{e}^{2 \varphi} g_{0}, K$ la courbure de Gauss de $g$, et $\mathcal{N} \mathrm{d} v=\mathcal{N}(t, x, y) d v$ le noyau de Schwartz de l'opérateur $\mathrm{e}^{-t A}$, nous avons

$$
\mathcal{N}(t, x, x)=\frac{1}{4 \pi t}+\left(\frac{K}{12 \pi}-\frac{V e^{-2 \varphi}}{4 \pi}\right)+O(t), \quad \text { quand } t \rightarrow 0^{+} .
$$

Nous en déduisons immédiatement le corollaire suivant :

Corollaire 3.2. - Soit $\left(\mathcal{M}, g_{0}\right)$ une surface riemannienne compacte sans bord. Soit aussi $\psi$ une fonction $C^{\infty}$ sur $\mathcal{M}$ à valeurs dans $\mathbb{R}$, et $V_{0}$ et $V_{1}$ deux potentiels positifs non nuls sur $\mathcal{M}$. Alors

$$
\ln \frac{\operatorname{det}_{\zeta} e^{-2 \psi}\left(\Delta_{g_{0}}+V_{1}\right)}{\operatorname{det}_{\zeta} \mathrm{e}^{-2 \psi}\left(\Delta_{g_{0}}+V_{0}\right)}=\ln \frac{\operatorname{det}_{\zeta}\left(\Delta_{g_{0}}+V_{1}\right)}{\operatorname{det}_{\zeta}\left(\Delta_{g_{0}}+V_{0}\right)}+\frac{1}{2 \pi} \int_{\mathcal{M}} \psi\left(V_{1}-V_{0}\right) \mathrm{d} v_{0},
$$

où $\mathrm{d} v_{0}$ est la mesure volume associée à $g_{0}$.

Nous en arrivons à la démonstration du théorème 1.1.

BULLETIN DE LA SOCIÉtÉ MATHÉMATIQUE DE FRANCE 
Proposition 3.3. - Soit $(\mathcal{M}, g)$ une variété riemannienne compacte dont le groupe d'isométries agit de manière transitive sur $\mathcal{M}$ et $m$ une constante stritement positive. Alors,

$$
\left.\frac{\mathrm{d}}{\mathrm{d} t}\right|_{t=0} \zeta_{\Delta_{g}+m+t V}(s)=-s v_{0} \zeta_{\Delta_{g}+m}(s+1) .
$$

Démonstration. - D'après la formule de variation des fonctions $\zeta$ (voir [8], p. $75-77)$ :

$$
\frac{\mathrm{d}}{\mathrm{d} t} \zeta_{A_{t}}(s)=-s \operatorname{Trace}_{L_{2}}\left(\left(\frac{\mathrm{d}}{\mathrm{d} t} A_{t}\right) \circ A_{t}^{-(s+1)}\right) .
$$

Si $\left(\varphi_{n}, \lambda_{n}\right)_{n \in \mathbb{N}}$ est une résolution spectrale de $\Delta_{g}$, nous en déduisons :

$$
\begin{aligned}
\left.\frac{\mathrm{d}}{\mathrm{d} t}\right|_{t=0} \zeta_{\Delta_{g}+m+t V}(s) & =-s \operatorname{Trace}_{L_{2}}\left(\mathcal{V} \circ\left(\Delta_{g}+m\right)^{-s-1}\right) \\
& =-\frac{1}{\Gamma(s)} \int_{0}^{+\infty} t^{s} \int_{\mathcal{M}} V(x) \sum_{n \geq 0} \mathrm{e}^{-\left(\lambda_{n}+m\right) t} \varphi_{n}^{2}(x) \mathrm{d} v_{g}(x) \mathrm{d} t .
\end{aligned}
$$

La démonstration suivante fait suite à une communication orale de G. Carron : étant donnée l'hypothèse sur le groupe d'isométries de $g$, la fonction

$$
x \in \mathcal{M} \longmapsto K(t, x, x)=\sum_{n \in \mathbb{N}} \mathrm{e}^{-\lambda_{n} t} \varphi_{n}^{2}(x)
$$

est indépendante de $x$, et donc

$$
\int_{\mathcal{M}} V(x) \sum_{n \geq 0} \mathrm{e}^{-\left(\lambda_{n}+m\right) t} \varphi_{n}^{2}(x) \mathrm{d} v_{g}(x)=v_{0} \operatorname{Trace}_{L_{2}}\left(\mathrm{e}^{-t\left(\Delta_{g}+m\right)}\right) .
$$

Ainsi,

$$
\left.\frac{\mathrm{d}}{\mathrm{d} t}\right|_{t=0} \zeta_{\Delta_{g}+m+t V}(s)=-\frac{v_{0}}{\Gamma(s)} \int_{0}^{+\infty} t^{s} \operatorname{Trace}_{L_{2}}\left(\mathrm{e}^{-t\left(\Delta_{g}+m\right)}\right),
$$

dont on déduit le résultat grâce à $\Gamma(s+1)=s \Gamma(s)$.

Demonstration du théorème 1.1. - Supposons pour commencer que $(\mathcal{M}, g)$ soit une variété riemannienne compacte sans bord telle que le groupe d'isométries de $g$ agisse de manière transitive sur $\mathcal{M}$. Soit $V$ un potentiel positif non constant. Posons à nouveau $A_{t}:=\Delta_{g}+v_{0}+t\left(V-v_{0}\right)$, où $v_{0}$ est défini dans le théorème. La fonction

$$
t \in[0,1] \longmapsto \psi(t)=\ln \operatorname{det}_{\zeta} A_{t}
$$

est strictement concave et, d'après la proposition 3.3 , pour tout $t$ dans $[0,1]$ et tout $s \in \mathbb{C},\left.\frac{\mathrm{d}}{\mathrm{d} t}\right|_{t=0} \zeta_{A_{t}}(s)=0$. En particulier, $\psi^{\prime}(0)=\left.\left.\frac{\mathrm{d}}{\mathrm{d} t}\right|_{t=0} \frac{\mathrm{d}}{\mathrm{d} s}\right|_{s=0} \zeta_{A_{t}}=0$.

La fonction $\psi$ est donc strictement concave sur $I$ et sa dérivée s'annule en 0 . Par conséquent, $\psi(0)>\psi(1)$; ce qui est équivalent à

$$
\operatorname{det}_{\zeta}\left(\Delta_{g}+V\right)<\operatorname{det}_{\zeta}\left(\Delta_{g}+v_{0}\right)
$$

TOME $134-2006-\mathrm{N}^{\mathrm{O}} 3$ 
Nous avons donc démontré le cas 1) du théorème, ainsi que le cas 2) lorsque la courbure de $g$ est constante (ces métriques ont en effet un groupe d'isométrie transitif).

Soit maintenant $\mathcal{M}=S^{2}$ ou $S^{1} \times S^{1}$. D'après le théorème d'uniformisation, il existe une unique fonction $\varphi$ de classe $C^{\infty}$ sur $\mathcal{M}$ telle que $g_{0}=\mathrm{e}^{2 \varphi} g$ ait une courbure constante et même volume que $g$. Alors $\Delta_{g_{0}}=\mathrm{e}^{-2 \varphi} \Delta_{g}$. Ainsi, grâce au corollaire 3.2 ,

(5) $\ln \frac{\operatorname{det}_{\zeta}\left(\Delta_{g}+V\right)}{\operatorname{det}_{\zeta}\left(\Delta_{g}+v_{0} \mathrm{e}^{2 \varphi}\right)}=\ln \frac{\operatorname{det}_{\zeta}\left(\Delta_{g_{0}}+V \mathrm{e}^{-2 \varphi}\right)}{\operatorname{det}_{\zeta}\left(\Delta_{g_{0}}+v_{0}\right)}-\frac{1}{2 \pi} \int_{\mathcal{M}} \varphi\left(V \mathrm{e}^{-2 \varphi}-v_{0}\right) \mathrm{d} v_{0}$.

Or, $g_{0}$ a un groupe d'isométries transitif et $\int_{\mathcal{M}} v_{0} \mathrm{~d} v_{g_{0}}=\int_{\mathcal{M}} V \mathrm{e}^{-2 \varphi} \mathrm{d} v_{g_{0}}$. La première partie de la démonstration du théorème 1.1 implique donc la deuxième.

\section{Le théorème $\mathbf{1 . 3}$}

Nous identifierons notre tore riemannien $(\mathcal{M}, g)$ à $\left(\mathbb{R}^{2} / \Lambda, \mathrm{e}^{2 \varphi}\left(\mathrm{dx}^{2}+\mathrm{dy}^{2}\right)\right)$ dans la suite, où $\Lambda=\mathbb{Z} \oplus \mathbb{Z} r \mathrm{e}^{i \theta} ;(\theta, r)$ sera choisi dans $\left[\frac{1}{2} \pi, \frac{1}{3} \pi\right] \times[1,+\infty[$ et $\varphi: \mathbb{R}^{2} \rightarrow \mathbb{R}$ sera $C^{\infty}$ et $\Lambda$-périodique. Enfin, $(\vec{\imath}, \vec{\jmath})$ sera une base orthonormée de $\mathbb{C}$ et $\vec{u}_{\theta}$ le vecteur d'affixe $\mathrm{e}^{i \theta}$.

Dans un premier temps, nous supposerons que $g$ est plate, i.e. que $\varphi$ est l'application nulle. Nous traiterons le cas général dans la section 4.3.3.

Choix d'une suite de triangulations. - Définissons une suite de graphes $\left(G_{n}\right)_{n \geq 3}$, qui s'identifient canoniquement à des triangulations de $\mathcal{M}$. Nous noterons $S_{n}$ l'ensemble de ses sommets et $E_{n}$ l'ensemble de ses arêtes.

- Graphe (figure 1). - Soit le graphe $\Lambda_{n}^{\prime}$, dont les sommets sont les points de $n^{-1} \Lambda$, et les arêtes sont définies ainsi : pour tout couple $(i, j) \in \mathbb{Z}^{2}$, l'ensemble des coordonnées dans la base $\left(n^{-1} \vec{\imath}, n^{-1} \vec{u}_{\theta}\right)$ des voisins du sommet de coordonnées $(i, j)$ est $\{(i, j-1) ;(i, j+1) ;(i+1, j) ;(i-1, j) ;(i+1, j-1) ;(i-1, j+1)\}$. Posons alors

$$
G_{n}:=\Lambda_{n}^{\prime} / \Lambda
$$

- Plongement. - Cette construction fournit une application injective canonique $j_{n}: G_{n} \hookrightarrow \mathbb{R}^{2} / \Lambda$ où les arêtes de $G_{n}$ s'identifient à des segments de $\mathbb{R}^{2} / \Lambda$, et les faces à des triangles euclidiens isométriques deux à deux. Nous noterons $\mathbb{R}^{S_{n}}$ l'ensemble $\left\{f: S_{n} \rightarrow \mathbb{R}\right\}$. Cet espace vectoriel s'injecte canoniquement dans $L^{2}(\mathcal{M})$ par l'application de Whitney

$$
W_{n}: \mathbb{R}^{S_{n}} \longrightarrow H^{1}(\mathcal{M}) \longleftrightarrow L^{2}(\mathcal{M})
$$

qui consiste à prolonger $f \in \mathbb{R}^{S_{n}}$ en une fonction affine sur chaque triangle.

BULletin DE LA SOCIÉtÉ MATHÉMATIQUE DE FRANCE 


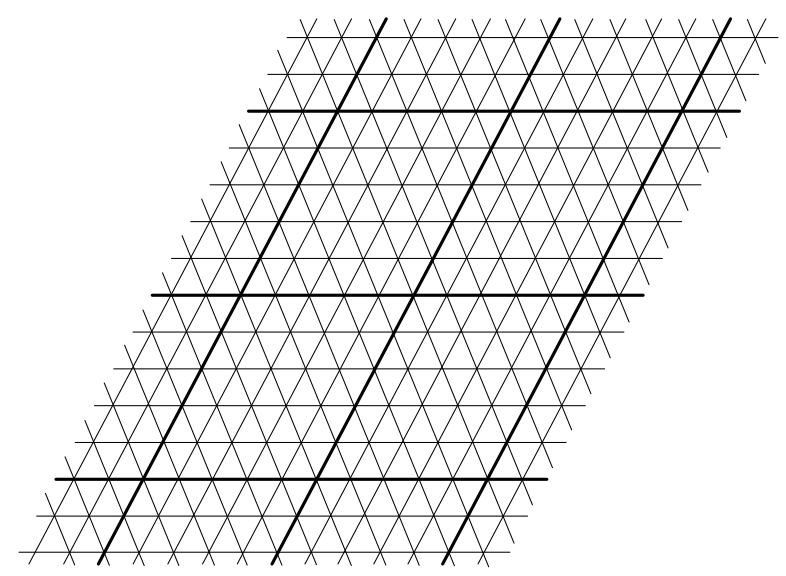

Figure 1. Triangulation $G_{5}$

- Produit scalaire. - Nous définissons un produit scalaire sur $\mathbb{R}^{S_{n}}$ par

$$
(f, g) \in\left(\mathbb{R}^{S_{n}}\right)^{2} \longmapsto(f \mid g):=\frac{r \sin \theta}{n^{2}} \sum_{v \in S_{n}} f(v) g(v) .
$$

- Énergie de Dirichlet. - $W_{n}\left(\mathbb{R}^{S_{n}}\right)$ étant inclus dans $H^{1}$, nous prendrons la restriction à $W_{n}\left(\mathbb{R}^{S_{n}}\right)$ de l'énergie de Dirichlet donnée par la métrique $g$ sur $\mathcal{M}$, qui s'écrit

$$
q_{n}: f \in \mathbb{R}^{S_{n}} \longmapsto \int_{\mathcal{M}}\left|\nabla W_{n}(f)\right|^{2} \mathrm{~d} v_{g}(x) .
$$

L'opérateur $\Delta_{n} \in \operatorname{End}\left(\mathbb{R}^{S_{n}}\right)$ est alors défini par l'égalité suivante, valable pour toute $f \in \mathbb{R}^{S_{n}}$ :

$$
q_{n}(f)=\left(\Delta_{n} f \mid f\right) .
$$

Lorsqu'il s'agira de discrétiser un opérateur de Schrödinger $A:=\Delta_{g}+V$, nous choisirons, pour tout $n \in \mathbb{N}$,

$$
A_{n}:=\Delta_{n}+p_{n}(V):\left(f \in \mathbb{R}^{S_{n}} \mapsto \Delta_{n}(f)+p_{n}(V) f\right),
$$

où $p_{n}: C^{0}(\mathcal{M}) \rightarrow \mathbb{R}^{S_{n}}$ est l'application restriction aux sommets du graphe, ce qui revient à considérer l'opérateur symétrique sur $\left(\mathbb{R}^{S_{n}},(. \mid).\right)$ associé à la forme quadratique

$$
f \in \mathbb{R}^{S_{n}} \longmapsto \int_{\mathcal{M}}\left|\nabla W_{n}(f)\right|^{2} \mathrm{~d} v_{g}(x)+\left(p_{n}(V) f \mid f\right) .
$$

TOME $134-2006-\mathrm{N}^{\mathrm{O}} 3$ 
Notations. - Les énergies de Dirichlet seront notées

$$
\Phi: h \in H^{1}(\mathcal{M}) \mapsto \int_{\mathcal{M}}|\nabla h| \mathrm{d} v_{g} \quad \text { et } \quad \Phi_{n}: h \in \mathbb{R}^{S_{n}} \mapsto \Phi\left(W_{n}(h)\right) .
$$

$V_{0}$ sera un potentiel positif $C^{\infty}$ sur $\mathcal{M}, v_{0}$ sa valeur moyenne pour la mesure volume $\mathrm{d} v_{g}, V:=V_{0}-v_{0}$ et nous noterons enfin $V_{t}:=v_{0}+t V$. Nous considèrerons les opérateurs et les déterminants associés :

$$
\begin{gathered}
A_{t}:=\Delta_{g}+v_{0}+t V \quad \text { et } \quad \psi: t \in I \mapsto \ln \operatorname{det}_{\zeta} A_{t}, \\
A_{t, n}:=\Delta_{n}+v_{0}+t V \quad \text { et } \quad \psi_{n}: t \in I \mapsto \ln \operatorname{det} A_{t, n} .
\end{gathered}
$$

Ils auront pour énergies correspondantes

$$
\begin{aligned}
q_{t, n} & : f \in \mathbb{R}^{S_{n}} \mapsto \Phi_{n}(f)+\left(p_{n}\left(V_{t}\right) f \mid f\right), \\
q_{t} & : f \in H^{1}(\mathcal{M}) \mapsto \Phi(f)+\int_{\mathcal{M}}\left(V_{t} f^{2}(x)\right) \mathrm{d} v_{g}(x)
\end{aligned}
$$

Nous choisirons d'utiliser la même lettre $\mathcal{V}$ pour les opérateurs

$$
\begin{aligned}
& \mathcal{V}:=\frac{\mathrm{d}}{\mathrm{d} t} A_{t}:\left(f \in L^{2}(\mathcal{M}) \mapsto V f \in L^{2}(\mathcal{M})\right) \text { et } \\
& \mathcal{V}:=\frac{\mathrm{d}}{\mathrm{d} t} A_{t, n}:\left(f \in \mathbb{R}^{S_{n}} \mapsto p_{n}(V) f \in \mathbb{R}^{S_{n}}\right) .
\end{aligned}
$$

Pour tout vecteur tangent $X$ au-dessus de $x \in \mathcal{M}$, nous poserons

$$
|X|^{2}:=g_{x}(X, X)
$$

la norme dans $L^{2}\left(\mathrm{~d} v_{g}\right)$ sera notée $\|\cdot\|_{0}$ et le produit scalaire sur $\mathbb{R}^{S_{n}}\|\cdot\|$.

Le spectre des opérateurs $A_{t}$ et $A_{t, n}$ sera noté

$$
\sigma\left(A_{t}\right):=\left\{\lambda_{k, t}\right\}_{k \in \mathbb{N}^{*}} \quad \text { et } \quad \sigma\left(A_{t, n}\right):=\left\{\lambda_{k, t, n}\right\}_{1 \leq k \leq n^{2}} .
$$

Enfin, $\left\{\varphi_{k, t}\right\}_{k \in \mathbb{N}^{*}}$ sera une base $L^{2}$-orthonormée de fonctions propres de $A_{t}$ $\left(\varphi_{k, t}\right.$ étant associée à $\left.\lambda_{k, t}\right)$, et $\left\{\varphi_{k, t, n}\right\}_{1<k \leq n^{2}}$ sera une base (.|.)-orthonormée de fonctions propres de $A_{t, n}$. Comme il a été expliqué dans l'introduction, il s'agit de prouver les deux convergences (3), ce que nous ferons respectivement dans les sections 4.2 et 4.3 , après avoir diagonalisé l'opérateur $A_{0}=\Delta_{n}+v_{0}$. Enfin, dans la section 4.3.3, nous étudierons le cas où la métrique n'est pas plate.

4.1. Diagonalisation du laplacien. - Les coefficients de la matrice du laplacien dans la base canonique qui apparaissent dans la méthode des éléments finis lorsque la métrique est plate sont bien connus : ils s'écrivent pour toute fonction $f \in W_{n}\left(\mathbb{R}^{S_{n}}\right)$

$$
\int_{\mathcal{M}}|\nabla f|^{2}=\sum_{e=(i, j) \in E_{n}} c_{i j}(f(i)-f(j))^{2},
$$

où $c_{i j}=\frac{1}{2}(\cot (\alpha)+\cot (\beta)), \alpha$ et $\beta$ étant les deux angles opposés à l'arête $(i j)$ dans les deux triangles qui lui sont adjacents.

BULLETIN DE LA SOCIÉtÉ MATHÉMATIQUE DE FRANCE 
Dans le graphe que l'on considère, tous les triangles sont semblables. Le poids des arêtes horizontales est $c_{1}=\cot \alpha$, celui des arêtes « verticales » $c_{2}=\cot \beta$ et celui des arêtes obliques $c_{3}=\cot \theta$.

Dans toute la suite, nous ferons l'abus de notation qui consiste à identifier le couple $(k, \ell) \in \mathbb{Z}_{n}^{2}$ au sommet de $G_{n}$ qui correspond au point $n^{-1}\left(k+\ell r \mathrm{e}^{\mathrm{i} \theta}\right)+\Lambda$ de $\mathbb{R}^{2} / \Lambda$, et nous noterons $f_{k, \ell}$ la valeur de $f$ en ce sommet. L'opérateur $\Delta_{n}: \mathbb{R}^{S_{n}} \mapsto \mathbb{R}^{S_{n}}$ s'écrit alors, pour tout couple $(k, \ell)$ de $\mathbb{Z}_{n}^{2}$,

$$
\begin{aligned}
\left(\Delta_{n} f\right)_{k, \ell}=\frac{n^{2}}{r \sin \theta}[2( & \left.c_{1}+c_{2}+c_{3}\right) f_{k, \ell}-c_{1}\left(f_{k+1, \ell}+f_{k-1, \ell}\right) \\
& \left.-c_{2}\left(f_{k, \ell+1}+f_{k, \ell-1}\right)-c_{3}\left(f_{k-1, \ell+1}+f_{k+1, \ell-1}\right)\right],
\end{aligned}
$$

où le terme $n^{2} / r \sin \theta$ provient du choix du produit scalaire sur $\mathbb{R}^{S_{n}}$.

Il se trouve que dans le cadre où nous nous situons, les fonctions propres du graphe sont exactement les restrictions aux sommets du graphe des fonctions propres du laplacien sur le tore plat.

Soit $\Lambda^{*}:=\operatorname{Hom}(\Lambda, \mathbb{Z})$ l'ensemble des morphismes d'anneaux de $\Lambda$ dans $\mathbb{Z}$, i.e. le réseau dual de $\Lambda$. Cet ensemble $\Lambda^{*}$ s'identifie à un réseau de $\mathbb{R}^{2}$ via le produit scalaire canonique sur $\mathbb{R}^{2}$. Pour tout $\tau \in \Lambda^{*} / n \Lambda^{*}$, définissons $f_{\tau} \in \mathbb{R}^{S_{n}}$ par

$$
\forall(k, \ell) \in \mathbb{Z}_{n}^{2}, \quad\left(f_{\tau}\right)_{k, \ell}=\exp \left[\frac{2 i \pi}{n}\left(\tau, k \vec{\imath}+\ell r \vec{u}_{\theta}\right)\right]
$$

(ici, $\tau$ est identifié à l'affixe d'un de ses représentants de $\Lambda^{*}$ ).

Proposition 4.1. - La famille de fonctions $\left(1 / \sqrt{r \sin \theta} f_{\tau}\right)_{\tau \in \Lambda^{*} / n \Lambda^{*}}$ est une base orthonormée de vecteurs propres de $\left(\Delta_{n},(. \mid).\right)$ sur $\mathbb{R}^{S_{n}}$. Si on note $\lambda_{\tau, n}$ la valeur propre associée $\grave{a} f_{\tau}$, on a

$$
\lambda_{\tau, n}=\frac{4 n^{2}}{r \sin \theta}\left[c_{1} \sin ^{2} \frac{\pi(\tau, \vec{\imath})}{n}+c_{2} \sin ^{2} \frac{\pi\left(\tau, \overrightarrow{u_{\theta}}\right)}{n}+c_{3} \sin ^{2} \frac{\pi\left(\tau, \vec{\imath}-\overrightarrow{u_{\theta}}\right)}{n}\right] .
$$

4.2. La convergence des variations premières. - Puisque $\left.\frac{\mathrm{d}}{\mathrm{d} t}\right|_{t=0} \ln \operatorname{det} A_{t}$ est nulle (voir la preuve du théorème 1.1), la deuxième convergence de (3) est une conséquence du lemme suivant :

LEMME 4.2. - On a

$$
\left.\lim _{n \rightarrow \infty} \frac{\mathrm{d}}{\mathrm{d} t}\right|_{t=0} \ln \operatorname{det} A_{t, n}=0 .
$$

Démonstration. - Puisque $V_{1}$ est une fonction d'intégrale nulle, on montre en découpant sur chaque triangle que $\left|\left(V_{1} \mid 1\right)\right|=\mathcal{O}\left(\frac{1}{n}\right)$.

De plus, pour tout élément $\tau$ de $\Lambda^{*} / n \Lambda^{*}$, si l'on note $\left(v_{i}\right)_{1 \leq i \leq n^{2}}$ les sommets de $G_{n}$,

$$
\left(V_{1} f_{\tau} \mid f_{\tau}\right)=\frac{r \sin \theta}{n^{2}} \sum_{i=1}^{n^{2}} V_{1}\left(v_{i}\right) f_{\tau}\left(v_{i}\right) \bar{f}_{\tau}\left(v_{i}\right)=\left(V_{1} \mid 1\right)
$$

TOME $134-2006-\mathrm{N}^{\mathrm{O}} 3$ 
Ainsi,

$$
\left.\frac{\mathrm{d}}{\mathrm{d} t}\right|_{t=0} \ln \operatorname{det} A_{t, n}=\sum_{\tau \in \Lambda^{*} / n \Lambda^{*}} \frac{\left(V_{1} f_{\tau} \mid \bar{f}_{\tau}\right)}{\lambda_{\tau, n}+v_{0}}=\sum_{\tau \in \Lambda^{*} / n \Lambda^{*}} \frac{\left(V_{1} \mid 1\right)}{\lambda_{\tau, n}+v_{0}} .
$$

Et donc

$$
\begin{aligned}
\left|\frac{\mathrm{d}}{\mathrm{d} t}\right|_{t=0} \ln \operatorname{det} A_{t, n} \mid & \leq \sum_{p, \ell=0}^{n-1} \frac{|(V \mid 1)|}{\frac{4 n^{2}}{r \sin \theta}\left(c_{1} \sin ^{2} \frac{\pi p}{n}+c_{2} \sin ^{2} \frac{\pi \ell}{n}+c_{3} \sin ^{2} \frac{\pi(p-\ell)}{n}\right)+v_{0}} \\
& \leq \mathcal{O}\left(\frac{1}{n}\right) \sum_{0 \neq p^{2}+\ell^{2} \leq \frac{1}{2} n^{2}} \frac{1}{p^{2}+\ell^{2}} .
\end{aligned}
$$

Dans la somme apparaissent l'inverse des premières valeurs propres du tore plat $\mathbb{R}^{2} / \mathbb{Z}^{2}$. L'asymptotique de Weyl appliqué à cette surface nous permet alors d'affirmer que $\sum_{0 \neq p^{2}+\ell^{2} \leq \frac{1}{2} n^{2}} \frac{1}{p^{2}+\ell^{2}}=\mathcal{O}(\ln n)$, et donc que

$$
\left|\frac{\mathrm{d}}{\mathrm{d} t}\right|_{t=0} \ln \operatorname{det} A_{t, n} \mid \leq \mathcal{O}\left(\frac{\ln n}{n}\right) \underset{n \rightarrow \infty}{\longrightarrow} \mathrm{D}
$$

4.3. La convergence des variations secondes. - Nous montrons que les opérateurs de Schrödinger discrets obtenus par cette méthode des éléments finis donnent de bonnes approximations spectrales des opérateurs de Schrödinger définis sur la variété; c'est-à-dire que les spectres, les sous-espaces propres et les résolvantes discrets convergent, dans un sens propre à chacune de ces quantités, vers leurs analogues continus. La seule difficulté ici provient du fait que le produit scalaire sur le graphe ne coïncide pas avec la restriction de la norme $L^{2}$ de la variété.

Nous ne montrerons que des convergences simples en $t$ de ces quantités, notamment des dérivées secondes. C'est pourquoi dans un premier temps, et dans un souci de concision, nous ne ferons pas figurer l'indice $t$, que ce soit pour les opérateurs, les spectres, ou les fonctions propres. Le théorème de Montel et une borne sur les dérivées secondes nous permettront d'en conclure que ces dernières convergent uniformément.

\subsubsection{Approximations des énergies}

Lemme 4.3. - Soit $f \in \mathbb{R}^{S_{n}}$. Alors on a

$$
\left|\sqrt{\int_{\mathcal{M}} W_{n}(f)^{2}(x) \mathrm{d} x}-\sqrt{\frac{r \sin \theta}{n^{2}} \sum_{v \in S_{n}} f^{2}(v)}\right| \leq \frac{r+1}{n} \sqrt{\int_{\mathcal{M}}\left|\nabla W_{n}(f)\right|^{2} \mathrm{~d} v_{g}} .
$$

Démonstration. - Découpons autour de chaque sommet $v_{i}$ un parallélogramme $A_{i, n}$ centré en $v_{i}$, de telle manière que, pour tout $i$ différent de $j, A_{i, n}$ soit le translaté de $A_{j, n}$ de vecteur $v_{i}-v_{j}$, et que la réunion de ces parallélogrammes pavent le domaine fondamental de $\mathbb{R}^{2} / \Lambda$. Définissons une fonction $h$ constante par morceau en posant $h(x)=f(i)$, pour tout $x$ dans $A_{i, n}$. 
La fonction $h$ est définie presque partout sur $\mathcal{M}$ et appartient à $L^{2}(\mathcal{M})$. Soit $T_{i, n}$ une 2-cellule de la triangulation, et $x$ un point de $T_{i, n}$. Alors, puisque $x \mapsto \nabla W_{n}(f)(x)$ est constante dans l'intérieur de $T_{i, n}$ et $\operatorname{diam} T_{i, n} \leq(r+1) / n$,

$$
\int_{T_{i, n}}\left(h-W_{n}(f)\right)^{2}(x) \mathrm{d} v_{g}(x) \leq\left(\frac{r+1}{n}\right)^{2} \int_{T_{i, n}}\left|\nabla W_{n}(f)\right|^{2} \mathrm{~d} v_{g} .
$$

Ainsi,

$$
\begin{aligned}
& \left|\sqrt{\int_{\mathcal{M}} W_{n}(f)^{2}(x) \mathrm{d} x}-\sqrt{\frac{r \sin \theta}{n^{2}} \sum_{v \in S_{n}} f^{2}(v)}\right| \\
& =\left|\sqrt{\int_{\mathcal{M}}\left(W_{n}(f)\right)^{2}(x) \mathrm{d} x}-\sqrt{\int_{\mathcal{M}} h^{2}(x)}\right| \\
& \leq \sqrt{\int_{\mathcal{M}}\left(h-W_{n}(f)\right)^{2}} \leq \frac{r+1}{n} \sqrt{\int_{\mathcal{M}}\left|\nabla W_{n}(f)\right|^{2} \mathrm{~d} v_{g}} .
\end{aligned}
$$

LEMME 4.4. - Soit $T$ le triangle du plan complexe, dont les affixes des sommets sont $0,1, r \mathrm{e}^{i \theta}$. Soit aussi $f$ la restriction à $T$ d'une fonction de classe $C^{2}(U, \mathbb{R})$, où $U$ est un ouvert du plan contenant $T$, nulle en chacun des sommets du bord. Si on note $\epsilon$ le diamètre de $T, \alpha$ son aire et $M$ une borne sur la norme $L^{\infty}$ des dérivées partielles secondes de $f$, nous avons les estimations suivantes :

$$
\int_{T} f^{2}(x) \mathrm{d} x \leq \alpha \epsilon^{2}\|\nabla f\|_{\infty}^{2} \quad \text { et } \quad \int_{T}|\nabla f|^{2}(x) \mathrm{d} x \leq \frac{\alpha \epsilon^{2} M^{2}}{\sin ^{2} \frac{1}{2} \theta} .
$$

Démonstration. - La première estimation est immédiate et la deuxième vient de

$$
\left(\frac{\partial f}{\partial x_{1}}\right)^{2}+\left(\frac{\partial f}{\partial x_{2}}\right)^{2} \leq \frac{\epsilon^{2} M^{2}}{\sin ^{2} \frac{1}{2} \theta}
$$

Notons $p_{n}: C^{2}(\mathcal{M}) \rightarrow \mathbb{R}^{S_{n}}$ l'application restriction aux sommets du graphe. Ainsi, si $g$ est une fonction de classe $C^{2}, W_{n} \circ p_{n}(g)$ est la fonction de $W_{n}\left(\mathbb{R}^{S_{n}}\right)$ qui coïncide avec $g$ sur les sommets du graphe $G_{n}$ et que l'on étend de manière affine sur l'intérieur de chaque triangle. Ceux-ci sont tous isométriques et obtenus par une similitude de rapport $1 / n$ à partir du triangle dont les sommets ont pour affixes $1,0, r \mathrm{e}^{i \theta}$. Leur diamètre $\epsilon$ et leur aire $\alpha$ vérifient donc $\epsilon \leq(r+1) / n$ et $\alpha=r \sin \theta /\left(2 n^{2}\right)$. Nous noterons $f_{n}:=W_{n} \circ p_{n}(f)$.

Proposition 4.5. - Pour toutes constantes $K$ et $\epsilon$ positives, il existe un entier $n_{1}=n_{1}(K, \epsilon)$ tel que pour toute fonction $f$ continue sur $\mathcal{M}$, de norme $L^{2}$ égale à 1 , et $C^{2}$ en restriction à chaque 2 -cellule $T_{i, n}$ de la triangulation, on

TOME $134-2006-\mathrm{N}^{\mathrm{O}} 3$ 
ait les estimations suivantes dès que $\|f\|_{2, \infty} \leq K$ et $n \geq n_{1}$,

$$
\begin{aligned}
& \left|\int_{\mathcal{M}} f^{2}(x) \mathrm{d} v_{g}(x)-\left(p_{n}(f) \mid p_{n}(f)\right)\right| \leq \epsilon, \\
& \int_{\mathcal{M}}\left|\nabla\left(f-W_{n} \circ p_{n}(f)\right)\right|^{2} \mathrm{~d} v_{g} \leq \epsilon .
\end{aligned}
$$

Démonstration. - Ce sont des conséquences des lemmes 4.4, 4.3 et de l'inégalité

$$
\left|\int f^{2}-\left(p_{n}(f) \mid p_{n}(f)\right)\right| \leq\left|\int f^{2}-\int f_{n}^{2}\right|+\left|\int f_{n}^{2}-\left(p_{n}(f) \mid p_{n}(f)\right)\right| .
$$

La convergence des spectres et des sous-espaces propres discrets découleront de la proposition suivante :

Proposition 4.6. - Soient deux réels $K$ et $\epsilon$ strictement positifs. Il existe un entier $n_{0}$ dépendant de $K$ et de $\epsilon$ tel que pour tout entier $n$ plus grand que $n_{0}$, et toute fonction $f$ de classe $C^{2}$ sur $\mathcal{M}$, de norme $\|f\|_{2, \infty} \leq K$, et de norme $L^{2}$ égale à 1 ,

$$
\left|q(f)-\frac{q_{n}\left(p_{n}(f)\right)}{\left(p_{n}(f) \mid p_{n}(f)\right)}\right| \leq \epsilon .
$$

Dans le cas où $f \in W_{n}\left(\mathbb{R}^{S_{n}}\right)$, le résultat subsiste en remplaçant l'hypothèse $\|f\|_{2, \infty} \leq K$ par l'inégalité plus faible $\int_{\mathcal{M}}|\nabla f|^{2} \leq K$.

Démonstration. - Décomposons le membre de gauche de l'inégalité (8) de la façon suivante :

(9) $\quad q(f)-\frac{q_{n}\left(p_{n}(f)\right)}{\left(p_{n}(f) \mid p_{n}(f)\right)}$

$$
\begin{aligned}
=\frac{1}{\left(p_{n}(f) \mid p_{n}(f)\right)} & {\left[q(f)\left(\left(p_{n}(f) \mid p_{n}(f)\right)-1\right)+\int|\nabla f|^{2}\right.} \\
& \left.-\int\left|\nabla W_{n} \circ p_{n}(f)\right|^{2}+\int V f^{2}-\left(p_{n}(V f) \mid p_{n}(f)\right)\right] .
\end{aligned}
$$

Il suffit d'appliquer la proposition 4.5 et de remarquer que

$$
\begin{aligned}
\left.\left|\int_{\mathcal{M}}\right| \nabla f\right|^{2}-\int\left|\nabla W_{n} \circ p_{n}(f)\right|^{2} \mid \leq\left(\Phi\left(f-W_{n} \circ p_{n}(f)\right)\right)^{\frac{1}{2}}\left[(\Phi(f))^{\frac{1}{2}}\right. \\
\left.+\left(\Phi\left(W_{n} \circ p_{n}(f)\right)\right)^{\frac{1}{2}}\right] .
\end{aligned}
$$

Enfin, si $f \in W_{n}\left(\mathbb{R}^{S_{n}}\right)$, le lemme 4.3 fournit l'estimation manquante.

LEMme 4.7. - Il existe deux constantes $C_{1}$ et $C_{2}$ positives non nulles telles que, pour tout entier $n$ supérieur à 3 ,

$$
C_{1} k \leq \lambda_{k} \leq C_{2} k, \forall k \in \mathbb{N}^{*} \text { et } C_{1} k \leq \lambda_{k, n}, \forall 1 \leq k \leq n^{2} .
$$

BULLETIN DE LA SOCIÉtÉ MATHÉMATIQUE DE FRANCE 
Démonstration. - L'asymptotique de Weil $\lambda_{k}(A) \sim_{k} 2 \pi /\left(\operatorname{Vol}_{g}(\mathcal{M})\right) \cdot k$ et le fait que $\lambda_{1}(A)>0$ impliquent la première assertion.

Puisque $\left|\lambda_{k, n}-\lambda_{k}\left(\Delta_{n}\right)\right| \leq\|V\|_{\infty}$, il suffit de montrer que l'on a l'inégalité $\lambda_{k}\left(\Delta_{n}\right) \geq C_{1} k$. Si $F$ est un sous-espace vectoriel d'un espace de Hilbert $H$ et $q$ une forme quadratique sur $H$, alors les valeurs propres de $q_{\left.\right|_{H}}$ sont inférieures ou égales à celles de $q_{\mid F}$. D'où, d'après le principe du min-max :

$$
\lambda_{k}\left(\Delta_{g}\right) \leq \inf _{\substack{K \subset \mathbb{R}^{S_{n}} \\ \operatorname{dim} K=k}} \sup _{f \in H} \frac{\Phi_{n}(f)}{\int\left(W_{n}(f)\right)^{2}} .
$$

Or le lemme 4.3 implique l'existence d'une constante $C>0$ telle que pour $n$ suffisamment grand,

$$
\frac{1}{\int_{\mathcal{M}} W_{n}(f)^{2}} \leq \frac{C}{(f \mid f)}, \quad \forall f \in \mathbb{R}^{S_{n}} \backslash\{0\} .
$$

Alors $\lambda_{k}\left(\Delta_{g}\right) \leq C \lambda_{k}\left(\Delta_{n}\right)$.

\subsubsection{Convergences des quantités discrètes}

- Le spectre

Proposition 4.8. - Soit un entier $k$ non nul et un réel $\epsilon$ strictement positif. Il existe un rang $n_{0}$ dépendant de $k$ et de $\epsilon$ tel que $\left|\lambda_{k}-\lambda_{k, n}\right| \leq \epsilon$ pour tout $n$ supérieur à $n_{0}$

Nous avons besoin du

LEMME 4.9. - Fixons un entier naturel $k$ et $\left(\varphi_{i}\right)_{1 \leq i \leq k}$ une famille libre de fonctions propres associées aux $k$ premières valeurs propres de $A$. Si $G_{k}$ est l'espace vectoriel engendré par ces $k$ vecteurs, il existe un entier $n_{0}$ dépendant de $k$ tel que, pour tout $n$ supérieur à $n_{0}, \operatorname{dim} p_{n}\left(G_{k}\right)=\operatorname{dim} G_{k}=k$.

Démonstration du lemme 4.9. — L'opérateur différentiel $A^{2}$ est elliptique d'ordre 4 ; il existe donc d'après l'inégalité de Garding, une constante $C_{A}>0$ telle que, pour toute fonction $f$ dans $C^{2}(\mathcal{M})$,

$$
\|f\|_{2, \infty} \leq\|f\|_{4} \leq C_{A}\left(\|f\|_{0}+\left\|A^{2} f\right\|_{0}\right) .
$$

Soit alors $\varphi$ une fonction propre de $A$ associée à une valeur propre $\lambda$ inférieure à $\lambda_{k}$, de norme égale à 1 dans $L^{2}\left(\mathcal{M}, d v_{g}\right)$. Alors, $\|\varphi\|_{2, \infty} \leq C_{A}\left(1+\lambda_{k}^{2}\right)$.

Toute fonction $f$ dans la boule unité de $G_{k}$ pour la norme $L^{2}\left(\mathcal{M}, \mathrm{d} v_{g}\right)$ a donc aussi une norme $\|f\|_{2, \infty}$ majorée par une fonction de $k$. Or si $p_{n}: G_{k} \rightarrow \mathbb{R}^{S_{n}}$ n'est pas injective, il existe une fonction $\varphi$ de norme $L^{2}$ égale à 1 dans $G_{k}$ nulle en chaque sommet de $G_{n}$. Alors en tout point $x$ de $\mathcal{M}$, nous avons $|\varphi(x)| \leq$ $\operatorname{diam} \mathcal{O}(1 / n)\|\nabla \varphi\|_{\infty}$, ce qui est incompatible avec $\int_{\mathcal{M}} \varphi^{2}=1$.

TOME $134-2006-\mathrm{N}^{\mathrm{O}} 3$ 
Démonstration de la proposition 4.8. - Soit $\varphi \in G_{k}$ de norme 1. Dans la démonstration du lemme 4.9, nous avons prouvé l'existence d'une constante $C=C(k)$ telle que $\|\varphi\|_{2, \infty} \leq C$. D'après la proposition 4.6, il existe alors un entier $n_{0}=n_{0}(k, \epsilon)$ tel que pour $n$ plus grand que $n_{0}$,

$$
\left|\frac{q_{n}\left(p_{n}(\varphi)\right)}{\left(p_{n}(\varphi) \mid p_{n}(\varphi)\right)}-q(\varphi)\right| \leq \epsilon .
$$

Alors, pour $n$ supérieur à $n_{0}$,

$$
\lambda_{k, n}=\inf _{\substack{H \subset \mathbb{R}^{S_{n}} \\ \operatorname{dim} H=k}} \sup _{f \in H} \frac{q_{n}(f)}{(f \mid f)} \leq \sup _{\varphi \in G_{k}} \frac{q(\varphi)}{\|\varphi\|_{0}^{2}}+\epsilon \leq \lambda_{k}+\epsilon .
$$

Pour l'autre inégalité, rappelons que les valeurs propres d'une forme quadratique sont supérieures à celles de sa restriction à un sous-espace vectoriel. Si on note $\mu_{k, n}$ les valeurs propres de $f \in \mathbb{R}^{S_{n}} \mapsto q\left(W_{n}(f)\right)$ sur $\mathbb{R}^{S_{n}}$ muni de la norme euclidienne $f \mapsto \int W_{n}(f)^{2}$, on a $\lambda_{k} \leq \mu_{k, n}$. Mais

$$
\mu_{k, n}=\inf _{\substack{H \subset \mathbb{R}^{S_{n}} \\ \operatorname{dim} H=k}} \sup _{f \in H} \frac{q\left(W_{n}(f)\right)}{\int W_{n}(f)^{2}} \leq \sup _{f \in G_{k, n}} \frac{q\left(W_{n}(f)\right)}{\int W_{n}(f)^{2}},
$$

où $G_{k, n}=\operatorname{Vect}\left(\varphi_{1, n}, \ldots, \varphi_{k, n}\right)$, les $\varphi_{i, n}$ étant des fonctions propres, orthonormalisées pour le produit scalaire (.|.), associées aux $k$ premières valeurs propres de $A_{n}$. Or, les énergies de Dirichlet de ces fonctions vérifient

$$
\int_{\mathcal{M}}\left|\nabla W_{n}\left(\varphi_{i, n}\right)\right|^{2}=q_{n}\left(\varphi_{i, n}\right)-\left(V \varphi_{i, n} \mid \varphi_{i, n}\right) \leq\left(C_{2} k+\epsilon\right)+\|V\|_{\infty},
$$

et sont ainsi majorées par une fonction de $k$, indépendamment des valeurs de $n$. Nous pouvons donc appliquer ici aussi la proposition (4.6) : il existe $n_{0}=n_{0}(k, \epsilon)$ tel que pour $n \geq n_{0}$ et pour toute fonction $f$ dans $G_{k, n}$,

$$
\frac{q\left(W_{n}(f)\right)}{\int_{\mathcal{M}} W_{n}(f)^{2}} \leq \frac{q_{n}(f)}{(f \mid f)}+\epsilon
$$

et ainsi $\lambda_{k} \leq \mu_{k, n} \leq \sup _{f \in G_{k, n}} q_{n}(f) /(f \mid f)+\epsilon=\lambda_{k, n}+\epsilon$.

Nous regroupons dans un lemme deux estimations essentielles, portant sur des normes des fonctions propres, qui sont apparues au cours de la démonstration de la proposition 4.8 , et qui nous seront utiles par la suite :

LEMME 4.10. - Soit $k$ un entier positif. Il existe un réel $K=K(k)$ tel que, pour tout entier $n$,

1) pour toute fonction propre $\varphi \in L^{2}(\mathcal{M})$ de norme 1 de A, associée à l'une des $k$ premières valeurs propres de cet opérateur,

$$
\|\varphi\|_{2, \infty}^{2} \leq K
$$

BULletin DE LA SOCiÉtÉ MATHÉmATiQUe DE FRANCE 
2) pour toute fonction propre $\varphi \in \mathbb{R}^{S_{n}}$ de norme 1 de $A_{n}$, associée à l'une des $k$ premières valeurs propres de cet opérateur,

$$
\int_{\mathcal{M}}\left|\nabla W_{n}(\varphi)\right|^{2} d v_{g} \leq K
$$

Les fonctions propres. - Fixons un entier positif $k$ tel que $\lambda_{k+1}(A) \neq \lambda_{k}(A)$. Soit $5 \mu$ un minorant de tous les sauts de valeurs propres dans la suite des $k+1$ premières valeurs propres de $A$, i.e.

$$
0<5 \mu<\min \left\{\lambda_{i+1}(A)-\lambda_{i}(A) ; i \leq k \text { et } \lambda_{i+1}(A) \neq \lambda_{i}(A)\right\} .
$$

D'après la proposition 4.8 , il existe un entier $n_{0}=n_{0}(k)$ tel que, pour tout $n$ supérieur à $n_{0}$ et tout $i$ inférieur à $k+1$,

$$
\operatorname{dim} \operatorname{ker}\left(A-\lambda_{i} I\right)=\operatorname{dim} \bigoplus_{\left|\lambda-\lambda_{i}\right|<2 \mu} \operatorname{ker}\left(A_{n}-\lambda I\right) .
$$

Nous noterons alors

$$
G_{i}:=\operatorname{ker}\left(A-\lambda_{i} I\right) \subset C^{\infty}(\mathcal{M}), \quad G_{i, n}:=\bigoplus_{\left|\lambda-\lambda_{i}\right|<2 \mu} \operatorname{ker}\left(A_{n}-\lambda I\right) \subset \mathbb{R}^{S_{n}} .
$$

L'objectif est de prouver qu'à partir d'un certain rang $n_{1}$ dépendant de $k$ et de $\epsilon$, il existe pour tout $n$ supérieur à $n_{1}$ une base $(.,$.$) -orthonormée de$ $G_{i} \epsilon$-proche d'une base (.|.)-orthonormée de $G_{i, n}$. Nous prouvons pour cela que la projection $(.,$.$) -orthogonale sur G_{i}$ restreinte à $G_{i, n}$ est $\epsilon$-proche de l'identité (c'est le lemme 4.12), puis nous construisons grâce à cette projection une isométrie de $\left(G_{i, n},(.,).\right)$ dans $\left(G_{i},(. \mid).\right)$ également $\epsilon$-proche de l'identité (lemme 4.13).

Commençons par un lemme technique :

Lemme 4.11. - Soient $H$ un espace de Hilbert et $A: H \rightarrow H$ un opérateur autoadjoint positif à résolvante compacte. Soit $\left(\varphi_{k}, \lambda_{k}\right)_{k \in \mathbb{N}}$ une résolution spectrale de $A$, où $\left(\lambda_{k}\right)$ est une suite croissante de réels positifs. Soient $i, r \in \mathbb{N}^{*}$ et supposons

$$
\lambda_{i}=\cdots=\lambda_{i+r-1}<\lambda_{i+r}-5 \mu .
$$

Soit $x \in H$ de norme 1 , et posons

$$
q(x):=(A x, x)=\lambda_{i}+\alpha,
$$

où $|\alpha|<2 \mu$. Alors, si on note p la projection orthogonale sur $\operatorname{Vect}\left(\varphi_{i}, \ldots, \varphi_{i+r-1}\right)$, et $\Pi$ la projection orthogonale sur $\operatorname{Vect}\left(\varphi_{1}, \ldots, \varphi_{i-1}\right)$,

$$
\|p(x)-x\|^{2} \leq\left[\frac{\lambda_{i}+|\alpha|}{3 \mu}-1\right] \cdot\|\Pi(x)\|^{2}+\frac{|\alpha|}{3 \mu} .
$$

TOME $134-2006-\mathrm{N}^{\mathrm{O}} 3$ 
Démonstration. - Nous noterons $x_{k}:=\left(x, \varphi_{k}\right)$. On l'équivalence

$$
q(x)=\lambda_{i}+\alpha \Longleftrightarrow\left(\lambda_{i}+\alpha\right) \sum_{k=1}^{+\infty} x_{k}^{2}=\sum_{k=1}^{+\infty} \lambda_{k} x_{k}^{2} .
$$

D'où

$$
\sum_{k \geq i+r} x_{k}^{2} \leq \sum_{k \geq i+r} \frac{\lambda_{k}-\lambda_{i}-\alpha}{\lambda_{i+r}-\lambda_{i}-\alpha} x_{k}^{2}=\sum_{k \leq i+r-1} \frac{\lambda_{i}-\lambda_{k}+\alpha}{\lambda_{i+r}-\lambda_{i}-\alpha} x_{k}^{2} .
$$

Or $\lambda_{i+r}-\lambda_{i}-\alpha \geq 5 \mu-\alpha \geq 3 \mu$. Par conséquent,

$$
\begin{aligned}
1-\sum_{k \leq i+r-1} x_{k}^{2} & =\sum_{k \geq i+r} x_{k}^{2} \\
& \leq \frac{\lambda_{i}-\lambda_{1}+|\alpha|}{3 \mu} \sum_{k \leq i-1} x_{k}^{2}+\sum_{i \leq k \leq i+r-1} \frac{\lambda_{i}-\lambda_{k}+\alpha}{3 \mu} x_{k}^{2} \\
& \leq \frac{\lambda_{i}+|\alpha|}{3 \mu}\|\Pi(x)\|^{2}+\frac{|\alpha|}{3 \mu} \sum_{k=i}^{i+r-1} x_{k}^{2} .
\end{aligned}
$$

Et ainsi, $\|x-p(x)\|^{2}=1-\sum_{k=i}^{i+r-1} x_{k}^{2} \leq\left[\left(\lambda_{i}+|\alpha|\right) / 3 \mu-1\right] \cdot\|\Pi(x)\|^{2}+|\alpha| / 3 \mu$.

Lemme 4.12. - Pour tout $\epsilon$ strictement positif et inférieur à $\frac{1}{2}$ et tout entier $k$, il existe un entier $n_{1}=n_{1}(\epsilon, k)$ tel que, pour tout entier $n$ supérieur à $n_{1}$, tout entier $i$ inférieur à $k$ et tout $x$ dans $G_{i, n},\left\|p_{i}(x)-x\right\| \leq \epsilon\|x\|$, où $p_{i}$ est la projection orthogonale de $L^{2}(\mathcal{M})$ sur $G_{i}$.

Démonstration. - Nous procédons par récurrence sur les espaces $G_{i}$.

- Montrons le lemme pour $i=1$. D'après le théorème de Courant, la première valeur propre de $A$ est simple et, le graphe $G_{n}$ étant connexe, la première valeur propre de $A_{n}$ est également simple d'après le théorème de PerronFrobenius (voir [4]). Soit $\varphi_{1, n} \neq 0 \in G_{1, n}$. Il existe $n_{1}=n_{1}(\epsilon) \in \mathbb{N}$ tel que, pour $n \geq n_{1}$,

$$
\left|\frac{\left(A \varphi_{1, n}, \varphi_{1, n}\right)}{\left\|\varphi_{1, n}\right\|^{2}}-\lambda_{1}\right| \leq \epsilon
$$

En effet, $\left(\lambda_{1, n}\right)_{n \in \mathbb{N}}$ converge vers $\lambda_{1}$, et, d'après la proposition 4.6 et le lemme 4.10

$$
\left|\lambda_{1, n}-\frac{\left(A \varphi_{1, n}, \varphi_{1, n}\right)}{\left\|\varphi_{1, n}\right\|^{2}}\right|=\left|\frac{\left(A_{n} \varphi_{1, n} \mid \varphi_{1, n}\right)}{\left(\varphi_{1, n} \mid \varphi_{1, n}\right)}-\frac{\left(A \varphi_{1, n}, \varphi_{1, n}\right)}{\left\|\varphi_{1, n}\right\|^{2}}\right| \underset{n \rightarrow \infty}{\longrightarrow} 0 .
$$

En appliquant le lemme 4.11 à $x=\varphi_{1, n} /\left\|\varphi_{1, n}\right\|$ et $i=r=1$, on trouve $\left\|p_{1}(x)-x\right\| \leq \epsilon /(3 \mu)$.

- Supposons le lemme vrai pour tous les entiers $j$ inférieurs à $i$, et montronsle pour $i+1$. Nous choisissons un entier $n_{1}$ tel que pour $n$ supérieur à $n_{1}$, pour

BULLETIN DE LA SOCIÉtÉ MATHÉMATIQUE DE FRANCE 
tout $j \leq i$ et tout $y$ dans $G_{j, n},\left\|p_{j}(y)-y\right\| \leq(\epsilon / i)\|y\|$. En particulier, dès que $\epsilon / i<1$, les applications linéaires $p_{j}: G_{j, n} \rightarrow G_{j}$ sont bijectives et

$$
\left\|\left(p_{j}: G_{j, n} \rightarrow G_{j}\right)^{-1}\right\| \leq 1+2 \epsilon .
$$

Prenons maintenant $x$ dans $G_{i+1, n}$ de norme $L^{2}$ égale à 1 , et soit $\Pi$ la projection orthogonale sur $G_{1} \oplus \cdots \oplus G_{i}$. Alors,

$$
\|\Pi(x)\|^{2}=(x, \Pi(x)) \leq\|\Pi(x)-y\|+|(x, y)|,
$$

pour tout $y$ dans $L^{2}(\mathcal{M})$. Prenons pour $y=y_{1}+\cdots+y_{i}$ l'unique vecteur de $\bigoplus_{j \leq i} G_{j, n}$ qui vérifie $\Pi(x)=\sum_{j=1}^{i} p_{j}\left(y_{j}\right)$. Alors par hypothèse de récurrence,

$$
\|\Pi(x)-y\| \leq \sum_{j=1}^{i}\left\|p_{j}\left(y_{j}\right)-y_{j}\right\| \leq \epsilon \sup _{j}\left\|y_{j}\right\| .
$$

De plus, $\left\|y_{j}\right\|=\left\|\left(p_{j}: G_{j, n} \rightarrow G_{j}\right)^{-1} \circ p_{j} \circ \Pi(x)\right\| \leq\left\|\left(p_{j}: G_{j, n} \rightarrow G_{j}\right)^{-1}\right\| \leq 1+2 \epsilon$, d'après (11). Donc $\|\Pi(x)-y\| \leq 2 \epsilon$. Enfin, $x$ et $y$ appartiennent à la somme directe des sous-espaces propres associés aux $k$ premières valeurs propres de $A_{n}$. Leur énergies de Dirichlet sont donc bornées par une fonction de $k$, et quitte à augmenter $n_{1}$, pour $n$ supérieur à $n_{1}$, le lemme 4.3 nous donne l'inégalité $|(x, y)-(x \mid y)| \leq \epsilon$. Comme $(x \mid y)=0$, nous avons d'après (12),

$$
\|\Pi(x)\|^{2} \leq 2 \epsilon+\epsilon \leq 3 \epsilon .
$$

À nouveau, comme pour (10), et quitte à augmenter $n_{1}$,

$$
\left|\lambda_{i+1, n}-(A x, x)\right|=:|\alpha| \leq \epsilon .
$$

En utilisant (13) et (14), nous pouvons appliquer le lemme 4.11 à $x$, en posant $p:=p_{i+1}$ :

$$
\left\|p_{i+1}(x)-x\right\|^{2} \leq\left[3\left(\frac{\lambda_{i+1}+|\alpha|}{3 \mu}-1\right)+\frac{1}{3 \mu}\right] \boxminus
$$

LEMME 4.13. - Soient $G_{1}$ et $G_{2}$ deux sous-espaces vectoriels de même dimension d'un Hilbert $H$, tels que si $p_{1}: H \rightarrow G_{1}$ est la projection orthogonale sur $G_{1}$, on a pour tout $x$ dans $G_{2},\left\|p_{1}(x)-x\right\| \leq \epsilon\|x\|$, où $\epsilon \leq \frac{1}{2}$. Supposons de plus qu'il existe sur $G_{2}$ un produit scalaire (.|.) proche du produit hilbertien $(.,$.$) induit par l'inclusion G_{2} \subset H$, i.e. que pour tout $x, y$ dans $G_{2}$,

$$
|(x, y)-(x \mid y)| \leq \epsilon^{2} \sqrt{(x, x)} \cdot \sqrt{(y, y)} .
$$

Alors il existe une isométrie $U:\left(G_{2},(. \mid).\right) \rightarrow\left(G_{1},(.,).\right)$ et une constante universelle $c>0$ telles que pour tout $x$ dans $G_{2}$,

$$
\|U x-x\| \leq c \epsilon\|x\|,
$$

où \|. || est la norme du Hilbert $H$.

TOME $134-2006-\mathrm{N}^{\mathrm{O}} 3$ 
Démonstration. - L'application $p_{1}: G_{2} \rightarrow G_{1}$ est injective donc bijective. Nous restreindrons toujours $p_{1}$ à $G_{2}$, si bien que nous pourrons considérer l'opérateur $p_{1}^{-1}: G_{2} \rightarrow G_{1}$.

Il existe deux produits scalaires $(.,$.$) et (. \mid$.$) sur G_{2}$, donc deux adjoints de $\left(p_{1}: G_{2} \rightarrow G_{1}\right):$

$$
\begin{aligned}
& \left(p_{1}^{*}: G_{1} \rightarrow G_{2}\right):=\left(p_{1}:\left(G_{2},(. \mid .)\right) \rightarrow\left(G_{1},(., .)\right)\right)^{*}, \\
& \left(p_{2}: G_{1} \rightarrow G_{2}\right):=\left(p_{1}:\left(G_{2},(., .)\right) \rightarrow\left(G_{1},(., .)\right)\right)^{*} .
\end{aligned}
$$

Il est facile de se convaincre que $p_{2}$ est la restriction à $G_{1}$ de la projection orthogonale de $H$ sur $G_{2}$. Pour tout élément $x$ de $G_{2}$, nous poserons $\|x\|^{2}=(x, x)$ et $|x|^{2}=(x \mid x)$. De même, nous noterons $\|p\|$ la norme de l'opérateur $p$ induite par celle de $H$. Si en revanche, l'espace de départ ou l'espace d'arrivée de $p$ est $G_{2}$, et que l'on muni $G_{2}$ de $(. \mid$.$) , nous noterons |p|$ sa norme. Établissons quelques inégalités élémentaires utiles à cette démonstration :

1) $\left\|p_{1}^{-1}\right\| \leq(1-\epsilon)^{-1} \leq 1+2 \epsilon$.

2) $\left\|p_{2}(y)-y\right\| \leq\left\|p_{1}^{-1}(y)-y\right\| \leq \epsilon\left\|p_{1}^{-1}\right\|\|y\| \leq 2 \epsilon\|y\|$, pour tout $y$ dans $G_{1}$.

3) On a

$$
\left|p_{1}\right|^{2}=\sup _{x \in G_{2}} \frac{\left(p_{1}(x), p_{1}(x)\right)}{(x \mid x)} \leq \frac{1}{1-\epsilon^{2}} \sup _{x \in G_{2}} \frac{\left(p_{1}(x), p_{1}(x)\right)}{(x, x)} \leq \frac{1}{1-\epsilon^{2}} .
$$

Donc $\left|p_{1}\right| \leq 1+2 \epsilon$.

4) Pour tout $y$ dans $G_{1}$ et $x$ dans $G_{2},\left(p_{1}^{*}(y) \mid x\right)=\left(y, p_{1}(x)\right)=\left(p_{2}(y), x\right)$. Donc

$$
\begin{aligned}
\left(p_{1}^{*}(y)-p_{2}(y), x\right) & =\left|\left(p_{1}^{*}(y), x\right)-\left(p_{1}^{*}(y) \mid x\right)\right| \\
& \leq \epsilon^{2}\left\|p_{1}^{*}(y)\right\| \cdot\|x\| \leq \epsilon^{2}\left(1+\epsilon^{2}\right)\left|p_{1}^{*}(y)\right| \cdot\|x\| \\
& \leq \epsilon^{2}\left(1+\epsilon^{2}\right)(1+2 \epsilon)\|y\| \cdot\|x\| \leq c \epsilon^{2}\|y\| \cdot\|x\| .
\end{aligned}
$$

Ainsi, $\left\|p_{1}^{*}-p_{2}\right\|=\sup _{x, y}\left(p_{1}^{*}(y)-p_{2}(y), x\right) /(\|x\| \cdot\|y\|) \leq c \epsilon^{2}$.

5) Pour tout $y$ dans $G_{2}$,

$$
\begin{aligned}
\left\|\left(p_{1}^{-1}\right)^{*}(y)-y\right\| & \leq\left\|\left(p_{1}^{-1}\right)^{*}\right\| \cdot\left\|p_{1}^{*}(y)-y\right\| \\
& \leq\left(\left\|\left(p_{1}^{-1}\right)^{*}\right\|\right)\left(\left\|p_{1}^{*}(y)-p_{2}(y)\right\|+\left\|p_{2}(y)-y\right\|\right) \leq c \epsilon\|y\| .
\end{aligned}
$$

Notons $\sqrt{p_{1}^{*} \circ p_{1}}$ la racine carrée symétrique définie positive de $p_{1}^{*} \circ p_{1}$ : $G_{2},(. \mid.) \rightarrow G_{2},(. \mid$.$) , et$

$$
U:=\left(p_{1}^{-1}\right)^{*} \circ \sqrt{p_{1}^{*} \circ p_{1}}:\left(G_{2},(. \mid .)\right) \longrightarrow\left(G_{1},(., .)\right),
$$

l'opérateur unitaire qui apparaÓt dans la décomposition polaire de $p_{1}$. Soit $x$ dans $G_{2}$. Remarquons que

$$
U x-x=\left[\left(p_{1}^{-1}\right)^{*}\left(\sqrt{p_{1}^{*} \circ p_{1}}(x)\right)-\sqrt{p_{1}^{*} \circ p_{1}}(x)\right]+\sqrt{p_{1}^{*} \circ p_{1}}(x)-x .
$$

BULletin DE LA SOCiÉtÉ MATHÉmATiQUe DE FRANCE 
Or, $\sqrt{p_{1}^{*} \circ p_{1}}(x)-x=\left(p_{1}^{*} \circ p_{1}-\operatorname{Id}_{G_{2}}\right) \circ\left(\sqrt{p_{1}^{*} \circ p_{1}}+\operatorname{Id}_{G_{2}}\right)^{-1}(x)$. Par conséquent, si on pose $y:=\left(\sqrt{p_{1}^{*} \circ p_{1}}+\operatorname{Id}_{G_{2}}\right)^{-1}(x) \in G_{2}$,

$$
\begin{aligned}
\left\|\sqrt{p_{1}^{*} \circ p_{1}}(x)-x\right\| & =\left\|\left(p_{1}^{*}-p_{2}\right)\left(p_{1}(y)\right)+p_{2}\left(p_{1}(y)-y\right)+p_{2}(y)-y\right\| \\
& \leq c \epsilon\|x\| .
\end{aligned}
$$

De plus,

$$
\begin{aligned}
\left\|\left[\left(p_{1}^{-1}\right)^{*}\left(\sqrt{p_{1}^{*} \circ p_{1}}(x)\right)-\sqrt{p_{1}^{*} \circ p_{1}}(x)\right]\right\| & \leq 6 \epsilon\left\|\sqrt{p_{1}^{*} \circ p_{1}}(x)\right\| \\
& \leq 6 \epsilon(1+\epsilon)\left|\sqrt{p_{1}^{*} \circ p_{1}}(x)\right| \\
& \leq 6 \epsilon(1+\epsilon)\left|p_{1}\right| \cdot\|x\| \leq c \epsilon\|x\| .
\end{aligned}
$$

Enfin, grâce à (15), (16) et (17), nous obtenons $\|U x-x\| \leq c \epsilon\|x\|$.

Nous en arrivons enfin à la proposition qui établit la convergence des fonctions propres.

Proposition 4.14. - Soient $\epsilon$ un réel strictement positif, et $k_{0}$ un réel. Il existe alors $k \geq k_{0}$, un entier $n_{1}=n_{1}(\epsilon, k)$ et pour tout $n$ supérieur à $n_{1}$, deux familles orthonormées $\left(\varphi_{1, n}, \ldots, \varphi_{k, n}\right)$ et $\left(\varphi_{1}, \ldots, \varphi_{k}\right)$ de fonctions propres respectivement de $A_{n}$ et de $A$ telles que pour tout $i \leq k$

$$
\left\|\varphi_{i}-W_{n}\left(\varphi_{i, n}\right)\right\|_{0} \leq \epsilon
$$

Démonstration. - Soit $k$ le plus petit entier supérieur à $k_{0}$ tel que $\lambda_{k+1} \neq \lambda_{k}$. Comme au début de cette section, fixons $5 \mu$ un minorant de tous les sauts de valeurs propres dans la suite des $k$ premières valeurs propres de $A$. D'après la proposition 4.8, il existe un entier $n_{0}=n_{0}(k, \mu)$ tel que pour tout $n$ supérieur à $n_{0}$ et tout $i$ inférieur à $k,\left|\lambda_{i}-\lambda_{i, n}\right| \leq 2 \mu$. Ainsi pour un tel couple d'entiers $(i, n)$,

$$
\operatorname{dim} \operatorname{ker}\left(A-\lambda_{i} I\right)=\operatorname{dim} \bigoplus_{\left|\lambda-\lambda_{i}\right|<2 \mu} \operatorname{ker}\left(A_{n}-\lambda I\right) .
$$

Posons alors $G_{i}:=\operatorname{ker}\left(A-\lambda_{i} I\right)$ et $G_{i, n}:=\bigoplus_{\left|\lambda-\lambda_{i}\right|<2 \mu} \operatorname{ker}\left(A_{n}-\lambda I\right)$. D'après les lemmes 4.12 et 4.13 , il existe un entier $n_{1}$ et une isométrie $U_{i, n}: G_{i, n} \rightarrow G_{i}$ tels que pour tout $n$ supérieur à $n_{1}$, tout entier $i$ inférieur à $k$ et tout $x$ dans $G_{i, n}$, $\left\|U_{i, n}(x)-x\right\| \leq c \epsilon\|x\|$. Ainsi, il suffit pour conclure de choisir une base orthonormée $\left(\varphi_{i, n}, \ldots, \varphi_{i+r, n}\right)$ de $G_{i, n}$ constituée de fonctions propres de $A_{n}$ et de poser, pour tout $0 \leq j \leq r$,

$$
\varphi_{i+j}:=U_{i, n}\left(\varphi_{i+j, n}\right) .
$$

- Les résolvantes

Proposition 4.15. - Soit $\Pi_{n}$ la projection orthogonale sur $W_{n}\left(\mathbb{R}^{S_{n}}\right)$ et $\varphi$ une fonction de classe $C^{2}$ sur $\mathcal{M}$. Alors

$$
\left\|A^{-1}(\varphi)-A_{n}^{-1} \circ \Pi_{n}(\varphi)\right\|_{0} \underset{n \rightarrow \infty}{\longrightarrow} 0 .
$$

TOME $134-2006-\mathrm{N}^{\mathrm{O}} 3$ 
Démonstration. - Soit $\epsilon$ un réel strictement positif. Fixons $k_{0} \in \mathbb{N}$ tel que $\sum_{j \geq k_{0}} 1 / \lambda_{j, n}^{2} \leq \epsilon$, et $\sum_{j \geq k_{0}} 1 / \lambda_{j}^{2} \leq \epsilon$, pour tout entier $n \in \mathbb{N}$ (ceci est rendu possible par les minorations du lemme 4.7). D'après les propositions 4.8 et 4.14 , il existe un entier $k$ supérieur à $k_{0}$, un rang $n_{1} \in \mathbb{N}$ et deux familles orthonormées $\left(\varphi_{1}, \ldots, \varphi_{k}\right)$ et $\left(\varphi_{1, n}, \ldots, \varphi_{k, n}\right)$ de fonctions propres associées aux $k$ premières valeurs propres respectivement de $A$ et de $A_{n}$ tels que, pour tout $n$ supérieur à $n_{1}$ et tout entier $j \leq k,\left|\lambda_{j, n}-\lambda_{j}\right| \leq \epsilon$ et $\left\|\varphi_{j, n}-\varphi_{j}\right\| \leq \epsilon$.

Nous complétons la famille $\left(\varphi_{1}, \ldots, \varphi_{k}\right)$ en une base orthonormée $\left(\varphi_{j}\right)_{j \in \mathbb{N}^{*}}$ de $L^{2}(\mathcal{M})$ de fonctions propres de $A$, et la famille $\left(\varphi_{1, n}, \ldots, \varphi_{k, n}\right)$ en une base orthonormée $\left(\varphi_{j, n}\right)_{1 \leq j \leq n^{2}}$ de $\mathbb{R}^{S_{n}}$ de fonctions propres de $A_{n}$. Alors, quelque soit $\varphi \in L^{2}$ de norme 1 ,

$$
A^{-1}(\varphi)=\sum_{j=1}^{+\infty} \frac{\left(\varphi_{j}, \varphi\right)}{\lambda_{j}} \varphi_{j} \quad \text { et } \quad A_{n}^{-1} \circ \Pi_{n}(\varphi)=\sum_{j=1}^{\alpha_{n}} \frac{\left(\varphi_{j, n} \mid \Pi_{n}(\varphi)\right)}{\lambda_{j, n}} \varphi_{j, n} .
$$

Les produits scalaires dans ces deux sommes diffèrent : (.,.) est le produit Hilbertien de $L^{2}\left(\mathcal{M}, \mathrm{d} v_{g}\right)$ et $(. \mid$.$) est le produit scalaire sur \mathbb{R}^{S_{n}}$. D'où si

$$
(I):=\left\|\sum_{j \leq k} \frac{\left(\varphi_{j}, \varphi\right)}{\lambda_{j}} \varphi_{j}-\frac{\left(\varphi_{j, n} \mid \Pi_{n}(\varphi)\right)}{\lambda_{j, n}} \varphi_{j, n}\right\|,
$$

alors $\left\|\left[A^{-1}-A_{n}^{-1} \circ \Pi_{n}\right](\varphi)\right\| \leq(I)+2 \epsilon$. Or,

$$
\begin{aligned}
(I) & \leq \sum_{j \leq k}\left|\frac{\left(\varphi_{j}, \varphi\right)}{\lambda_{j}}\right| \cdot\left\|\varphi_{j}-\varphi_{j, n}\right\|+\left|\frac{\left(\varphi_{j}, \varphi\right)}{\lambda_{j}}-\frac{\left(\varphi_{j, n} \mid \Pi_{n}(\varphi)\right)}{\lambda_{j, n}}\right| \\
& \leq \sum_{j \leq k} \frac{\epsilon}{\lambda_{1}}+\left|\frac{\left(\varphi_{j}, \varphi\right)}{\lambda_{j}}-\frac{\left(\varphi_{j, n} \mid \Pi_{n}(\varphi)\right)}{\lambda_{j, n}}\right| .
\end{aligned}
$$

Puisque $g:(x, y) \in[-1,1] \times\left[\frac{1}{2} \lambda_{1}, \lambda_{k}+1\right] \mapsto x / y$, est uniformément continue, il suffit d'obtenir $\left|\left(\varphi_{j}, \varphi\right)-\left(\varphi_{j, n} \mid \Pi_{n}(\varphi)\right)\right| \leq \epsilon$ pour tout $n$ assez grand. Cette dernière égalité s'obtient en écrivant

$$
\begin{aligned}
\left(\varphi_{j}, \varphi\right)-\left(\varphi_{j, n} \mid \Pi_{n}(\varphi)\right)=\left(\varphi_{j}-\varphi_{j, n}, \varphi\right)+\left(\varphi_{j, n}, \varphi-\Pi_{n}(\varphi)\right) \\
+\left(\varphi_{j, n}, \Pi_{n}(\varphi)\right)-\left(\varphi_{j, n} \mid \Pi_{n}(\varphi)\right) .
\end{aligned}
$$

En effet, $\left\|\varphi-\Pi_{n}(\varphi)\right\|_{0} \leq\left\|\varphi-W_{n} \circ p_{n}(\varphi)\right\|_{0}$, et $\left|\left(\varphi_{j, n}, \Pi_{n}(\varphi)\right)-\left(\varphi_{j, n} \mid \Pi_{n}(\varphi)\right)\right| \leq \epsilon$ d'après le lemme 4.3.

- Les dérivées secondes du logarithme du déterminant

Fixons une norme $\|\cdot\|_{s}$ sur chaque espace de Sobolev $H^{s}$, et notons $\|.\|_{s, u}$ les normes quélles induisent sur l'espace des opérateurs linéaires continus de $H^{s}$ dans $H^{u}$. Nous reprenons les notations avec les indices $t$.

Lemme 4.16. - Pour tout réel $t$ dans [0,1], la norme induite de l'opérateur $A_{t, n} \circ \mathcal{V}_{n} \circ A_{t, n}^{-1}$ agissant sur l'espace euclidien $\left(\mathbb{R}^{S_{n}},(. \mid).\right)$, est majorée par une constante $C_{3}$ indépendante de $n$. 
Démonstration. — C'est une conséquence du résultat suivant :

LEMME 4.17. - Soit $\mathbb{R}^{\alpha}$ muni du produit scalaire canonique, dont nous noterons ||.|| la norme associée. Soit A une matrice symétrique définie positive dont les coefficients diagonaux sont strictement positifs et les coefficients non diagonaux sont négatifs ou nuls, et $V$ une matrice diagonale. Alors, si l'on note

$$
\|\cdot\|: B \in \operatorname{End}\left(\mathbb{R}^{\alpha}\right) \mapsto \max _{x \in \mathbb{R}^{\alpha}} \frac{\|B x\|}{\|x\|}, \quad\|\cdot\|_{\infty}: B \in \operatorname{End}\left(\mathbb{R}^{\alpha}\right) \mapsto \max _{1 \leq i, j \leq \alpha}\left|B_{i, j}\right|,
$$

(où les $B_{i, j}$ sont les coefficients de la matrice $B$ ), nous avons

$$
\left\|A \circ V \circ A^{-1}\right\| \leq\|V\|_{\infty}\left(2\|A\|_{\infty} \cdot\left\|A^{-1}\right\|+1\right) .
$$

En effet, dans le cas qui nous intéresse, $\left\|A_{t, n}^{-1}\right\|=1 / \lambda_{1, t, n}$ converge vers $\lambda_{1, t}^{-1}$, les hypothèses sur la négativité des coefficients de $A_{n, t}$ sont vérifiées grâce au fait que c'est un opérateur de Schrödinger, et $\left\|A_{n, t}\right\|_{\infty} \leq$ $\max _{1 \leq i, j \leq \alpha_{n}}\left\{\left|\Delta_{n}\right|_{i, j}\right\}+\left\|V_{t}\right\|_{\infty}$ est également majoré indépendamment de $n$.

Démonstration du lemme 4.17. — Soit $\lambda>\|A\|_{\infty}$ et $t>0$. L'exponentielle

$$
\mathrm{e}^{-t A}=\mathrm{e}^{-t \lambda} \sum_{n \geq 0} \frac{\left[t\left(-A+\lambda I_{\alpha}\right)\right]^{n}}{n !}
$$

est une matrice à coefficients positifs. D'où $A^{-1}=\int_{0}^{+\infty} \mathrm{e}^{-t A} \mathrm{~d} t$ est également à coefficients strictement positifs. Si $B=\left[b_{i j}\right]_{i, j}$, nous noterons $|B|$ la matrice $\left[\left|b_{i j}\right|\right]_{i, j}$. On montre facilement que $\|B\| \leq\||B|\|$, et que si $B$ et $C-B$ sont deux matrices à coefficients tous positifs, alors $\|B\| \leq\|C\|$.

Posons $A=\left[a_{i j}\right], A^{-1}=\left[b_{i j}\right], V=\operatorname{Diag}\left(V_{1}, \ldots, V_{\alpha}\right)$ et $C=\left[c_{i j}\right]=A V A^{-1}$. Alors, pour tout $(i, j) \in\left(\mathbb{R}^{\alpha}\right)^{2}$,

$$
\begin{aligned}
\left|c_{i j}\right| & =\left|\sum_{k=1}^{\alpha} a_{i k} V_{k} b_{k j}\right| \leq-\sum_{k \neq i}^{\alpha} a_{i k}\left|V_{k}\right| b_{k j}+a_{i i}\left|V_{i}\right| b_{i j} \\
& \leq\|V\|_{\infty}\left[2\|A\|_{\infty} A^{-1}+I_{\alpha}\right]_{i j} .
\end{aligned}
$$

Ainsi $\left\|A V A^{-1}\right\| \leq\||C|\| \leq\|V\|_{\infty}\left[2\|A\|_{\infty} \cdot\left\|A^{-1}\right\|+1\right]$.

Proposition 4.18. - On a

$$
\sup _{t \in[0 ; 1]}\left|\psi_{n}^{\prime \prime}(t)-\psi^{\prime \prime}(t)\right| \underset{n \rightarrow \infty}{\longrightarrow} 0 \text {. }
$$

Démonstration. - Soit un réel $k \geq k_{0}$, un entier $n_{1}=n_{1}\left(\epsilon, t, k_{0}\right)$ tels que pour tout $n \geq n_{1}$, il existe des bases orthonormées de vecteurs propres associées aux $k$ premières valeurs propres de $A_{t}$ et $A_{n, t}$, telles que $\left\|\varphi_{j, t}-W_{n}\left(\varphi_{j, n, t}\right)\right\|_{0} \leq \epsilon$

TOME $134-2006-\mathrm{N}^{\mathrm{O}} 3$ 
et $\left|\lambda_{j, t}-\lambda_{j, n, t}\right| \leq \epsilon$, pour tout $j \leq k$. Dans ces bases, une fois complétées, nous avons d'après la proposition 2.3,

$$
\begin{aligned}
& \psi^{\prime \prime}(t)=-\sum_{j=1}^{\infty}\left(\mathcal{V} \circ A_{t}^{-1} \circ \mathcal{V} \circ A_{t}^{-1}\left(\varphi_{j, t}\right), \varphi_{j, t}\right), \\
& \psi_{n}^{\prime \prime}(t)=-\sum_{j=1}^{n^{2}}\left(\mathcal{V} \circ A_{t, n}^{-1} \circ \mathcal{V} \circ A_{t, n}^{-1}\left(\varphi_{j, n, t}\right) \mid \varphi_{j, n, t}\right) .
\end{aligned}
$$

- Nous allons prouver la convergence simple en $t$. Fixons $t$ dans $[0,1]$ et $\epsilon$ un réel strictement positif. Il existe $k_{0}$ tel que $\sum_{j \geq k_{0}} C_{3}\|V\|_{\infty} /\left(C_{1}^{2} j^{2}\right) \leq \epsilon$, où $C_{3}$ et $C_{1}$ sont deux constantes dont l'existence nous est donnée respectivement par les lemmes 4.16 et 4.7. Soit alors $k \geq k_{0}$,

$$
\begin{aligned}
\left|\sum_{j=k}^{\alpha_{n}}\left(\mathcal{V} \circ A_{t, n}^{-1} \circ \mathcal{V} \circ A_{t, n}^{-1}\left(\varphi_{j, n, t}\right) \mid \varphi_{j, n, t}\right)\right| \\
=\left|\sum_{j \geq k} \frac{\left(A_{t, n} \circ \mathcal{V} \circ A_{t, n}^{-1}\left(V \varphi_{j, t, n}\right) \mid \varphi_{j, t, n}\right)}{\lambda_{j, t, n}^{2}}\right| \leq \epsilon .
\end{aligned}
$$

On obtient de même l'inégalité $\left|\sum_{j=k}^{\infty}\left(\mathcal{V} \circ A_{t}^{-1} \circ \mathcal{V} \circ A_{t}^{-1}\left(\varphi_{j, t}\right), \varphi_{j, t}\right)\right| \leq \epsilon$ en remarquant que $A_{t} \circ \mathcal{V} \circ A_{t}^{-1}: C^{\infty}(\mathcal{M}) \rightarrow C^{\infty}(\mathcal{M})$ est un opérateur pseudodifférentiel d'ordre 0 et s'étend donc en un endomorphisme continu sur $L^{2}(\mathcal{M})$. D'après (19), nous obtenons

$$
\begin{aligned}
\mid \psi_{n}^{\prime \prime}(t) & -\psi^{\prime \prime}(t) \mid \\
& \leq 2 \epsilon+\sum_{j=1}^{k}\left|\frac{\left(A_{t}^{-1}\left(V \varphi_{j, t}\right), V \varphi_{j, t}\right)}{\lambda_{j, t}}-\frac{\left(A_{t, n}^{-1}\left(V \varphi_{j, t, n}\right) \mid V \varphi_{j, t, n}\right)}{\lambda_{j, t, n}}\right| .
\end{aligned}
$$

Posons $0<m^{-1}=\min _{n \geq n_{1}}\left\{\lambda_{1, t}, \lambda_{1, n, t}\right\}$. Alors on a $\left\|A_{t}^{-1}\right\|_{0,0} \leq m$ et $\left\|A_{t, n}^{-1}\right\| \leq m$. À nouveau, grâce à la continuité uniforme de la fonction $(x, y) \in$ $\left[-m\|V\|_{\infty}^{2}, m\|V\|_{\infty}^{2}\right] \times\left[m^{-1}, \lambda_{k, t}+1\right] \mapsto x / y$, il suffit de montrer dans la somme (21) la proximité des numérateurs. Décomposons la différence $\left|\left(A_{t, n}^{-1}\left(V \varphi_{j, n, t}\right) \mid V \varphi_{j, n, t}\right)-\left(A_{t}^{-1}\left(V \varphi_{j, t}\right), V \varphi_{j, t}\right)\right| \leq(\mathrm{I})+(\mathrm{II})+(\mathrm{III})+(\mathrm{IV})+(\mathrm{V})$, où

$$
\begin{aligned}
(\mathrm{I}) & =\left|\left(A_{t, n}^{-1}\left(V \varphi_{j, n, t}\right) \mid V \varphi_{j, n, t}-\Pi_{n}\left(V \varphi_{j, t}\right)\right)\right|, \\
(\mathrm{II}) & =\left|\left(A_{t, n}^{-1}\left(V \varphi_{j, n, t}-\Pi_{n}\left(V \varphi_{j, t}\right)\right) \mid \Pi_{n}\left(V \varphi_{j, t}\right)\right)\right|, \\
(\mathrm{III}) & =\left|\left(A_{t, n}^{-1} \circ \Pi_{n}\left(V \varphi_{j, t}\right) \mid \Pi_{n}\left(V \varphi_{j, t}\right)\right)-\left(A_{t, n}^{-1} \circ \Pi_{n}\left(V \varphi_{j, t}\right), \Pi_{n}\left(V \varphi_{j, t}\right)\right)\right|, \\
(\mathrm{IV}) & =\left|\left(\left(A_{t, n}^{-1} \circ \Pi_{n}-A_{t}^{-1}\right)\left(V \varphi_{j, t}\right), \Pi_{n}\left(V \varphi_{j, t}\right)\right)\right|, \\
(\mathrm{V}) & =\left|\left(A_{t}^{-1}\left(V \varphi_{j, t}\right), V \varphi_{j, t}-\Pi_{n}\left(V \varphi_{j, t}\right)\right)\right| .
\end{aligned}
$$

BULletin DE LA SOCiÉtÉ MATHÉMATIQUE DE FRANCE 
Alors (I) $\leq\left\|A_{t, n}^{-1}\left(V \varphi_{j, n, t}\right)\right\| \cdot\left\|\Pi_{n}\left(V \varphi_{j, t}\right)-V \varphi_{j, n, t}\right\|$. Puisque les énergies de Dirichlet des fonctions $\left\{\Pi_{n}\left(V \varphi_{j, t}\right)\right.$ et $\left.V \varphi_{j, n, t}, n \in \mathbb{N}\right\}$ sont uniformément bornées, nous pouvons supposer grâce au lemme 4.3, que

$$
\left\|\Pi_{n}\left(V \varphi_{j, t}\right)-V \varphi_{j, n, t}\right\| \leq(1+\epsilon)\left\|\Pi_{n}\left(V \varphi_{j, t}\right)-W_{n}\left(V \varphi_{j, n, t}\right)\right\|_{0} .
$$

Donc,

$$
\begin{aligned}
&(\mathrm{I}) \leq m\|V\|_{\infty}\left[\left\|V \varphi_{j, t}-V W_{n}\left(\varphi_{j, n, t}\right)\right\|_{0}\right. \\
&\left.+\left\|V W_{n}\left(\varphi_{j, n, t}\right)-W_{n}\left(V \varphi_{j, n, t}\right)\right\|_{0}\right](1+\epsilon) \\
& \leq m\|V\|_{\infty}\left[\|V\|_{\infty} \epsilon+\left\|V W_{n}\left(\varphi_{j, n, t}\right)-W_{n}\left(V \varphi_{j, n, t}\right)\right\|_{0}\right](1+\epsilon) .
\end{aligned}
$$

Or, il est facile de se convaincre, en décomposant $W_{n}\left(V \varphi_{j, n, t}\right)$ et $V W_{n}\left(\varphi_{j, n, t}\right)$ sur chaque 2 -cellule $T_{p, n}$ de la triangulation que

$\left\|V W_{n}\left(\varphi_{j, n, t}\right)-W_{n}\left(V \varphi_{j, n, t}\right)\right\|_{0} \leq \operatorname{Vol}_{g}(\mathcal{M})\|V\|_{\infty} \max _{p}\left(\operatorname{diam}\left(T_{p, n}\right)\right)\left\|\varphi_{j, n, t}\right\|_{\infty}$.

De même,

$$
\begin{aligned}
\text { (II) } & \leq m\left\|\Pi_{n}\left(V \varphi_{j, t}\right)-V \varphi_{j, n, t}\right\| \cdot\left\|V \varphi_{j, t}\right\| \\
& \leq m\|V\|_{\infty}\left\|\Pi_{n}\left(V \varphi_{j, t}\right)-W_{n}\left(V \varphi_{j, n, t}\right)\right\|_{0}(1+\epsilon)^{2} \\
& \leq m\|V\|_{\infty}\left(\|V\|_{\infty}+1\right) \epsilon(1+\epsilon)^{2} .
\end{aligned}
$$

De plus,

$(\mathrm{V}) \leq m\|V\|_{\infty} \cdot\left\|V \varphi_{j, t}-\Pi_{n}\left(V \varphi_{j, t}\right)\right\|_{0} \leq m\|V\|_{\infty} \cdot\left\|V \varphi_{j, t}-W_{n} \circ p_{n}\left(V \varphi_{j, t}\right)\right\|_{0}$.

Pour (IV), cela provient de la convergence forte des inverses discrètes.

Pour (III), d'après le lemme 4.3 , il suffit de prouver que $\Pi_{n}\left(V \varphi_{j, t}\right)$ et $A_{t, n}^{-1}$ 。 $\Pi_{n}\left(V \varphi_{j, t}\right)$ ont des énergies de Dirichlet bornées par une fonction dépendant de $k . \Pi_{n}\left(V \varphi_{j, t}\right)$ converge pour la norme $H^{1}$ vers $V \varphi_{j, t}$, son énergie de Dirichlet est donc bornée par une fonction de $k$. Posons $g_{n}:=A_{t, n}^{-1} \circ \Pi_{n}\left(V \varphi_{j, t}\right)$. Alors

$$
\Delta_{n} g_{n}+V_{t} g_{n}=A_{t, n} g_{n}=\Pi_{n}\left(V \varphi_{j, t}\right),
$$

ce qui implique

$$
\begin{aligned}
\int_{\mathcal{M}}\left|\nabla g_{n}\right|^{2} & =\left(\Delta_{n} g_{n} \mid g_{n}\right)=\left(\Pi_{n}\left(V \varphi_{j, t}\right) \mid g_{n}\right)-\left(V_{t} g_{n} \mid g_{n}\right) \\
& \leq\left(\left\|\Pi_{n}\left(V \varphi_{j, t}\right)\right\|+\left\|V_{t}\right\|_{\infty} \cdot\left\|g_{n}\right\|\right)\left\|g_{n}\right\| .
\end{aligned}
$$

Or, $\left\|g_{n}\right\| \leq m\left\|\Pi_{n}\left(V \varphi_{j, t}\right)\right\|$ est bornée par une fonction de $k$. D'où la convergence simple de $\psi_{n}^{\prime \prime}$ vers $\psi^{\prime \prime}$.

- Prouvons maintenant la convergence uniforme. Les fonctions $f_{n}^{\prime \prime}$ et $f^{\prime \prime}$ sont en fait holomorphes sur un voisinage $U$, d'adhérence compacte, de $[0,1]$ dans $\mathbb{C}$. Nous avons besoin du lemme suivant :

TOME $134-2006-\mathrm{N}^{\mathrm{O}} 3$ 
LEMME 4.19. - Il existe une constante $C$ telle que pour tout entier $n$ positif,

$$
\sup _{t \in U}\left|\psi_{n}^{\prime \prime}(t)\right| \leq C \text {. }
$$

Différons la démonstration de ce lemme afin d'achever celle de la proposition $4.18:\left(\psi_{n}^{\prime \prime}\right)_{n \in \mathbb{N}}$ est une suite bornée de fonctions holomorphes sur $U$. D'après le théorème de Montel, cette suite est relativement compacte dans l'ensemble des fonctions holomorphes sur $U$ muni de la convergence uniforme sur tout compact de $U$. Or, cette suite ne possède qu'une seule valeur d'adhérence, puisque deux valeurs d'adhérence de $\left(\psi_{n}^{\prime \prime}\right)$ sont des fonctions holomorphes sur $U$ qui coïncident sur $[0,1]$. Toute suite incluse dans un compact qui ne possède qu'une seule valeur d'adhérence est une suite convergente, donc $\left(\psi_{n}^{\prime \prime}\right)_{n \in \mathbb{N}}$ converge uniformément sur tout compact vers $\psi^{\prime \prime}$. En particulier, $\sup _{t \in[0,1]}\left|\psi_{n}^{\prime \prime}-\psi^{\prime \prime}\right|$ tend vers zéro, ce qui achève la démonstration de la proposition 4.18 et donc celle du théorème 1.3 .

Démonstration du lemme 4.19. - Nous utilisons la norme trace. Pour tout endomorphisme $M$ sur $\mathbb{R}^{S_{n}}$, nous noterons $0 \leq \mu_{1}(M) \leq \mu_{2}(M) \leq \cdots \leq \mu_{n^{2}}(M)$ les valeurs propres de $|M|:=\sqrt{M^{*} M}$, appelées valeurs singulières de $M$. Nous utiliserons les estimations

$$
\mu_{k}(M N) \leq\|M\| \mu_{k}(N) \quad \text { et } \sum_{k=1}^{n^{2}} \mu_{k}(M N) \leq \sum_{k=1}^{\alpha} \mu_{k}(M) \mu_{k}(N),
$$

valables quels que soient $M, N \in \operatorname{End}\left(\mathbb{R}^{S_{n}}\right)$ (voir $[16$, th. 1.15, p. 12]). Ainsi,

$$
\left|\psi_{n}^{\prime \prime}(t)\right| \leq \operatorname{Trace}\left(\left|\mathcal{V}_{n} \circ A_{t, n}^{-1} \circ \mathcal{V}_{n} \circ A_{t, n}^{-1}\right|\right) \leq\|V\|_{\infty} \operatorname{Trace}\left(\left|A_{t, n}^{-1} \circ \mathcal{V}_{n} \circ A_{t, n}^{-1}\right|\right) \text {. }
$$

Or,

$$
\operatorname{Trace}\left(\left|A_{t, n}^{-1} \circ \mathcal{V}_{n} \circ A_{t, n}^{-1}\right|\right)=\sum_{k=1}^{n^{2}} \mu_{k}\left(A_{t, n}^{-1} \circ \mathcal{V}_{n} \circ A_{t, n}^{-1}\right) \leq\|V\|_{\infty}^{2} \sum_{k=1}^{\alpha_{n}}\left(\frac{1}{\lambda_{k, n, t}}\right)^{2}
$$

En appliquant le principe du min-max, nous pouvons finalement écrire

$$
\left|\psi_{n}^{\prime \prime}(t)\right| \leq\|V\|_{\infty}^{2} \sum_{k=1}^{+\infty}\left(\frac{1}{C_{1} k-\inf _{t \in[0,1]}\left\|V_{t}\right\|_{\infty}}\right)^{2}<+\infty
$$

4.3.3. Le cas d'une métrique quelconque. - Démontrons le théorème 1.3 dans le cas général. Soit $(\mathcal{M}, g)$ un tore de dimension 2 muni d'une métrique riemannienne. D'après le théorème d'uniformisation, il existe une métrique $g_{0}$ conforme à $g$, unique à homothétie près. Plus précisément, il existe un réseau $\Lambda=\mathbb{Z} 1 \oplus \mathbb{Z} r \mathrm{e}^{i \theta}$ de $\mathbb{C}$ et une fonction $C^{\infty} \varphi: \mathbb{R}^{2} / \Lambda \rightarrow \mathbb{R}$ telle que $(\mathcal{M}, g)$ est isométrique à $\left(\mathbb{R}^{2} / \Lambda, \mathrm{e}^{2 \varphi} g_{0}\right)$, où $g_{0}:=\mathrm{d} x^{2}+\mathrm{d} y^{2}$. Pour simplifier les notations, nous poserons $\mathcal{M}=\mathbb{R}^{2} / \Lambda$ et $g=\mathrm{e}^{2 \varphi} g_{0}$.

Soit $V_{0}$ un potentiel $C^{\infty}$ sur $\mathcal{M}$. Nous cherchons à discrétiser l'opérateur $\Delta_{g}+$ $V_{0}$. Nous reprenons pour cela la suite de triangulations $G_{n}=\left(V_{n}, E_{n}\right)$ associée

BULletin DE LA SOCiÉtÉ MATHÉmATiQUe DE FRANCE 
à $\mathbb{R}^{2} / \Lambda$ définie page 336 , ainsi que la forme quadratique $q_{n}: \mathbb{R}^{S_{n}} \rightarrow \mathbb{R}^{S_{n}}$ que nous noterons $q_{n, g_{0}}$. Comme structure euclidienne sur $\mathbb{R}^{S_{n}}$, nous prendrons, pour toute fonction $f \in \mathbb{R}^{S_{n}}$,

$$
(f \mid f)_{g}:=\frac{r \sin \theta}{n^{2}} \sum_{v \in S_{n}} \mathrm{e}^{2 \varphi(v)} f^{2}(v) .
$$

À nouveau, nous discrétisons $f \in L^{2} \mapsto V_{0} f \in L^{2}$ en prenant l'opérateur qui à tout vecteur $\delta_{v}$ de la base canonique, où $v \in S_{n}$, associe le vecteur $V_{0}(v) \delta_{v}$. Il est diagonal dans la base canonique. Quant à la suite de discrétisés $\Delta_{n, g}$ de $\Delta_{g}$, nous posons

$$
\Delta_{n, g}: f \in \mathbb{R}^{S_{n}} \longmapsto \mathrm{e}^{-2 \varphi} \Delta_{n, g_{0}}(f) \in \mathbb{R}^{S_{n}}
$$

où $\mathrm{e}^{-2 \varphi}$ est l'opérateur diagonal sur $\mathbb{R}^{S_{n}}$ défini par $\mathrm{e}^{-2 \varphi}\left(\delta_{v}\right)=\mathrm{e}^{-2 \varphi(v)} \delta_{v}$. L'opérateur $\Delta_{n, g}$ est l'opérateur symétrique sur $\left(\mathbb{R}^{S_{n}},(. \mid)_{g}\right)$ associé à la même énergie de Dirichlet que précédemment (nous traduisons ici l'invariance conforme de celle-ci).

Remarquons, même si nous ne l'utiliserons pas par la suite, que $g$ étant conforme à $g_{0}$, les angles de la triangulation sont les mêmes pour $g$ et $g_{0}$ : les valeurs propres discrètes $\lambda_{k}\left(\Delta_{n, g}\right)$ convergent donc lorsque $n$ tend vers l'infini vers $\lambda_{k}\left(\Delta_{g}\right)$, ce qui fait de $\left(\Delta_{n, g}+V_{0}\right)_{n \in \mathbb{N}}$ une suite raisonnable de discrétisations de $\Delta_{g}+V_{0}$.

Soient $V_{1}$ et $V_{0}$ deux potentiels positifs tels que $\int_{\mathcal{M}} V_{1} \mathrm{~d} v_{g}=\int_{\mathcal{M}} V_{0} \mathrm{~d} v_{g}$, et soient $A_{n, 1}:=\Delta_{n, g}+V_{1}$ et $A_{n, 0}:=\Delta_{n, g}+V_{0}$. On a

$$
\begin{aligned}
\frac{\operatorname{det} A_{n, 1}}{\operatorname{det} A_{n, 0}} & =\frac{\operatorname{det}\left(\mathrm{e}^{-2 \varphi} \Delta_{n, g_{0}}+V_{1}\right)}{\operatorname{det}\left(\mathrm{e}^{-2 \varphi} \Delta_{n, g_{0}}+V_{0}\right)} \\
& =\frac{\operatorname{det}\left(\Delta_{n, g_{0}}+\mathrm{e}^{2 \varphi} V_{1}\right)}{\operatorname{det}\left(\Delta_{n, g_{0}}+\mathrm{e}^{2 \varphi} V_{0}\right)} \underset{n \rightarrow \infty}{\longrightarrow} \frac{\operatorname{det}_{\zeta} \Delta_{g_{0}}+\mathrm{e}^{2 \varphi} V_{1}}{\operatorname{det}_{\zeta} \Delta_{g_{0}}+\mathrm{e}^{2 \varphi} V_{0}}
\end{aligned}
$$

Cette convergence est en effet une application du cas plat du théorème 1.3, démontré dans la section précédente, dès lors que l'on a vu que $\int_{\mathcal{M}} V_{1} \mathrm{e}^{2 \varphi} \mathrm{d} v_{g_{0}}=$ $\int_{\mathcal{M}} V_{0} \mathrm{e}^{2 \varphi} \mathrm{d} v_{g_{0}}$. De plus, d'après le corollaire 3.2 ,

$$
\begin{aligned}
\frac{\operatorname{det}_{\zeta} \Delta_{g_{0}}+\mathrm{e}^{2 \varphi} V_{1}}{\operatorname{det}_{\zeta} \Delta_{g_{0}}+\mathrm{e}^{2 \varphi} V_{0}} & =\frac{\operatorname{det}_{\zeta} \mathrm{e}^{2 \varphi}\left(\Delta_{g}+V_{1}\right)}{\operatorname{det}_{\zeta} \mathrm{e}^{2 \varphi}\left(\Delta_{g}+V_{0}\right)} \\
& =\frac{\operatorname{det}_{\zeta} \Delta_{g}+V_{1}}{\operatorname{det}_{\zeta} \Delta_{g}+V_{0}} \exp \left\{-\frac{1}{2 \pi} \int_{\mathcal{M}} \varphi\left(V_{1}-V_{0}\right) \mathrm{d} v_{g}\right\} .
\end{aligned}
$$

On conclut en voyant que si $K_{g}$ est la courbure de Gauss de la métrique $g=\mathrm{e}^{2 \varphi} g_{0}, K_{g}=\Delta_{g}(\varphi)$. 


\section{BIBLIOGRAPHIE}

[1] Burgheela (D.), Friedlander (L.) \& Kappeler (T.) - On the determinant of elliptic differential and finite difference operators in vector bundles over $S^{1}$, Comm. Math. Phys., t. 138 (1991), pp. 1-18.

[2] - On the determinant of elliptic boundary value problems on a line segment, Proc. Amer. Math. Soc., t. 123 (1995), pp. 3027-3038.

[3] Colin De Verdière (Y.) - Spectres de graphes, Société Mathématique de France, 1998.

[4] , Déterminants et intégrales de Fresnel, Ann. Inst. Fourier, t. 49 (1999), pp. 861-881.

[5] Duplantier (B.) \& DAVID (F.) - Exact partition functions and correlation functions of multiple Hamiltonian walks on the Manhattan lattice, J. Statist. Phys., t. 51 (1988), pp. 327-434.

[6] Forman (R.) - Functional determinants and geometry, Invent. Math., t. 88 (1987), pp. 447-493.

[7] , Determinants, finite-difference operators and boundary value problems, Comm. Math. Phys., t. 147 (1992), pp. 485-526.

[8] Gilkey (P. B.) - Invariance Theory, the Heat Equation, and the AtiyahSinger Index Theorem, 2nd ed., in Studies in Advanced Mathematics, 1994.

[9] Kenyon (R.) - The asymptotic determinant of the discrete laplacian, Acta Math., t. 185 (2000), pp. 239-286.

[10] Okikiolu (K.) - Critical metrics for the determinant of the Laplacian in odd dimensions, Ann. of Math., t. 153 (2001), pp. 471-531.

[11] Osgood (B.), Phillips (R.) \& Sarnak (P.) - Compact isospectral sets of surfaces, J. Funct. Anal., t. 80 (1988), pp. 212-234.

[12] _ Extremals of determinants of laplacians, J. Funct. Anal., t. 80 (1988), pp. 148-211.

[13] Pollicott (M.) \& Rocha (A. C.) - A remarkable formula for the determinant of the Laplacian, Invent. Math., t. 130 (1997), pp. 399-414.

[14] RAY (D. B.) \& Singer (I. M.) - R-Torsion and the Laplacian on Riemannian Manifolds, Advances Math., t. 7 (1971), pp. 145-210.

[15] Shubin (M. A.) - Pseudodifferential operators and spectral theory, Springer Verlag, 1980.

[16] Simon (B.) - Trace ideals and their applications, in Lecture Note Series, vol. 35, London Mathematical Society, Cambridge University Press, 1979. 